Steven A. Prescott, Stéphanie Ratté, Yves De Koninck and Terrence J. Sejnowski

J Neurophysiol 100:3030-3042, 2008. First published Oct 1, 2008; doi:10.1152/jn.90634.2008

You might find this additional information useful...

This article cites 48 articles, 28 of which you can access free at:

http://jn.physiology.org/cgi/content/full/100/6/3030\#BIBL

Updated information and services including high-resolution figures, can be found at:

http://jn.physiology.org/cgi/content/full/100/6/3030

Additional material and information about Journal of Neurophysiology can be found at: http://www.the-aps.org/publications/jn

This information is current as of December 5, 2008 . 


\title{
Pyramidal Neurons Switch From Integrators In Vitro to Resonators Under In Vivo-Like Conditions
}

\author{
Steven A. Prescott, ${ }^{1}$ Stéphanie Ratté, ${ }^{2}$ Yves De Koninck, ${ }^{3}$ and Terrence J. Sejnowski ${ }^{1,4}$ \\ ${ }^{1}$ Howard Hughes Medical Institute, Computational Neurobiology Laboratory, Salk Institute, La Jolla, California; ${ }^{2}$ Département de \\ Physiologie, Université de Montréal, Montreal, Quebec, Canada; ${ }^{3}$ Division de Neurobiologie Cellulaire, Centre de Recherche Université \\ Laval Robert-Giffard, Quebec, Quebec, Canada; and ${ }^{4}$ Division of Biological Sciences, University of California, San Diego, \\ La Jolla, California
}

Submitted 3 June 2008; accepted in final form 22 September 2008

Prescott SA, Ratté S, De Koninck Y, Sejnowski TJ. Pyramidal neurons switch from integrators in vitro to resonators under in vivolike conditions. J Neurophysiol 100: 3030-3042, 2008. First published October 1, 2008; doi:10.1152/jn.90634.2008. During wakefulness, pyramidal neurons in the intact brain are bombarded by synaptic input that causes tonic depolarization, increased membrane conductance (i.e., shunting), and noisy fluctuations in membrane potential; by comparison, pyramidal neurons in acute slices typically experience little background input. Such differences in operating conditions can compromise extrapolation of in vitro data to explain neuronal operation in vivo. For instance, pyramidal neurons have been identified as integrators (i.e., class 1 neurons according to Hodgkin's classification of intrinsic excitability) based on in vitro experiments but that classification is inconsistent with the ability of hippocampal pyramidal neurons to oscillate/resonate at theta frequency since intrinsic oscillatory behavior is limited to class 2 neurons. Using long depolarizing stimuli and dynamic clamp to reproduce in vivo-like conditions in slice experiments, we show that CA1 hippocampal pyramidal cells switch from integrators to resonators, i.e., from class 1 to class 2 excitability. The switch is explained by increased outward current contributed by the M-type potassium current $I_{\mathrm{M}}$, which shifts the balance of inward and outward currents active at perithreshold potentials and thereby converts the spike-initiating mechanism as predicted by dynamical analysis of our computational model. Perithreshold activation of $I_{\mathrm{M}}$ is enhanced by the depolarizing shift in spike threshold caused by shunting and/or sodium channel inactivation secondary to tonic depolarization. Our conclusions were validated by multiple comparisons between simulation and experimental data. Thus even so-called "intrinsic" properties may differ qualitatively between in vitro and in vivo conditions.

\section{N T R O D U C T I O N}

The high level of background synaptic input received by pyramidal neurons in the intact, awake brain can significantly alter the integrative properties of those neurons (Bernander et al. 1991; Destexhe and Paré 1999; Paré et al. 1998). Destexhe and Paré (1999) reported that, on average, background synaptic input to neocortical pyramidal neurons reduced input resistance by $80 \%$ while causing a $20 \mathrm{mV}$ depolarizing shift in membrane potential and a tenfold increase in the SD of voltage noise (for review, see Destexhe et al. 2003). Reducing input resistance (i.e., shunting) causes predictable shortening of both the membrane time and length constants;

Present address and address for reprint requests and other correspondence: S. A. Prescott, Dept. of Neurobiology, University of Pittsburgh, Biomedical Science Tower, W1455, 200 Lothrop St., Pittsburgh, PA 15213 (E-mail: prescott@neurobio.pitt.edu). this in turn causes the cell to behave more like a coincidence detector and less like an integrator insofar as the neuron responds more selectively to synchronized inputs (Bernander et al. 1991; Rudolph and Destexhe 2003). Thus passive membrane properties differ between in vitro and in vivo conditions with important consequences for how neurons process information. Noise caused by synaptic input has equally unintuitive implications, for instance, on firing rate modulation by shunting inhibition (Chance et al. 2002; Prescott and De Koninck 2003) and on contrast invariance of orientation tuning in visual cortex (Anderson et al. 2000). Wolfart et al. (2005) showed in thalamic neurons that conductance noise blurs the single-spike and burst firing modes (which are classically associated with depolarized and hyperpolarized membrane potentials, respectively) and interacts with intrinsic currents to determine the neuron's transfer function. Steriade (2001) described several examples in which noise influences "intrinsic" spiking patterns. Still other discrepancies are likely to exist between in vitro and in vivo conditions.

Here we address whether CA1 pyramidal neurons exhibit class 1 or class 2 excitability according to Hodgkin's classification scheme (Hodgkin 1948): class 1 neurons can maintain arbitrarily slow firing and therefore have a continuous frequencycurrent $(f-I)$ curve, whereas class 2 neurons cannot maintain firing below some critical rate and therefore have a discontinuous $f-I$ curve. Subsequent work has shown that this phenomenological difference derives from the different dynamical mechanisms responsible for spike initiation in each cell class (Izhikevich 2007; Prescott et al. 2008; Rinzel and Ermentrout 1998). The spike-initiating mechanism, in turn, has several important implications. For instance, the phase response curve (PRC) differs between class 1 and 2 neurons (Rinzel and Ermentrout 1998): a perturbation can only advance the next spike in class 1 neurons (i.e., monophasic PRC), whereas it can advance or delay the next spike in class 2 neurons depending on when the perturbation occurs (i.e., biphasic PRC). This property affects the synchronization of neurons within a network: class 1 neurons will not synchronize in a purely excitatory network, whereas class 2 neurons will (Ermentrout 1996; Hansel et al. 1995), although class 1 neurons can be synchronized by inhibitory input (e.g., Wang and Buzsáki 1996). Moreover, because of their spike-initiating mechanism, class 1 neurons summate inputs across a broad range of frequencies, whereas class 2 neurons have a preferred input frequency to which they

\footnotetext{
The costs of publication of this article were defrayed in part by the payment of page charges. The article must therefore be hereby marked "advertisement" in accordance with 18 U.S.C. Section 1734 solely to indicate this fact.
} 
resonate. Class 1 and 2 neurons have therefore been labeled integrators and resonators, respectively (Izhikevich 2007).

It seems to be commonly believed that pyramidal cells are class 1 , or at least that is how they have been modeled (e.g., Wilson 1999) and the assumption is implicit in the widespread use of leaky integrate-and-fire models. But there is an unrecognized discrepancy: hippocampal CA1 pyramidal neurons do indeed have continuous $f$ - $I$ curves according to in vitro experiments (e.g., Gustafsson and Wigstrom 1981), consistent with class 1 excitability, but they can (under slightly different conditions) also exhibit subthreshold oscillations and resonance at $3-10 \mathrm{~Hz}$ or $\theta$ frequency (Hu et al. 2002; Leung and $\mathrm{Yu}$ 1998; Leung and Yim 1991; Pike et al. 2000), consistent with class 2 excitability. Needless to say, network oscillations are important for brain function (Buzsáki 2006) and although intrinsic resonance is not required for entrainment of pyramidal cell activity, it influences responsiveness to oscillatory input and is likely to affect $\theta$-related phase precession (Harris et al. 2002). It is therefore critical that we understand and resolve this discrepancy so that neurons are modeled with the appropriate spike-initiating mechanism.

We reasoned that synaptic bombardment experienced in vivo causes a relative increase in outward current via shunting and activation or inactivation of currents secondary to tonic depolarization. Based on our recent work on spiking initiating dynamics (Prescott et al. 2008), a relative increase in outward current could theoretically convert neuronal excitability from class 1 to class 2 . In other words, CA1 pyramidal cells that behave as integrators under in vitro conditions may behave as resonators under in vivo conditions. To investigate this, we first tested the effects of shunting and adaptation in a computational model amenable to dynamical analysis. Then, directed by specific predictions derived from modeling, we tested CA1 pyramidal neurons under different in vivo-like conditions. As we predicted, results demonstrate that "intrinsic" spike-initiating dynamics do indeed differ between in vitro and in vivo conditions because of the relative increase in outward current in vivo.

\section{METHODS}

\section{Slicing and electrophysiology}

All experiments were done in accordance with regulations of the Canadian Council on Animal Care and have been described by us previously (Prescott et al. 2006). Adult male Sprague Dawley rats were anesthetized with intraperitoneal injection of sodium pentobarbital $(30 \mathrm{mg} / \mathrm{kg})$ and perfused intracardially with ice-cold oxygenated $\left(\begin{array}{lll}95 \% & \mathrm{O}_{2}-5 \% & \mathrm{CO}_{2}\end{array}\right)$ sucrose-substituted artificial cerebrospinal fluid (ACSF) containing (in $\mathrm{mM}$ ) 252 sucrose, $2.5 \mathrm{KCl}, 2 \mathrm{CaCl}_{2}, 2 \mathrm{MgCl}_{2}$, 10 glucose, $26 \mathrm{NaHCO}_{3}, 1.25 \mathrm{NaH}_{2} \mathrm{PO}_{4}$, and 5 kynurenic acid. The brain was rapidly removed and sectioned coronally to give $400-\mu \mathrm{m}$ thick slices, which were kept in normal oxygenated ACSF (126 mM $\mathrm{NaCl}$ instead of sucrose and without kynurenic acid) at room temperature until recording.

Slices were transferred to a recording chamber constantly perfused with oxygenated $\left(95 \% \quad \mathrm{O}_{2}-5 \% \quad \mathrm{CO}_{2}\right)$ ACSF heated to $30-31^{\circ} \mathrm{C}$. Pyramidal neurons in the CA1 region of hippocampus were recorded in the whole cell configuration with $>80 \%$ series resistance compensation using an Axopatch 200B amplifier (Molecular Devices; Palo Alto, CA). Only data from regular spiking pyramidal cells judged healthy on the basis of three criteria (resting membrane potential less than $-50 \mathrm{mV}$, spikes overshooting $0 \mathrm{mV}$, and input resistance $>100$
$\mathrm{M} \Omega$ ) were included in analysis presented here. Average resting $V$ was $-73 \pm 1(\mathrm{SE}) \mathrm{mV}$, and average input resistance was $159 \pm 9 \mathrm{M} \Omega$. Variations in resting $V$ were eliminated by adjusting $V$ to $-70 \mathrm{mV}$ through tonic current injection. Reported values of $V$ were corrected for liquid junction potential calculated (see Barry and Lynch 1991) to be $9 \mathrm{mV}$ based on the intracellular recording solution, which contained (in $\mathrm{mM}$ ) $135 \mathrm{KMeSO}_{4}, 5 \mathrm{KCl}, 10 \mathrm{HEPES}$, and $2 \mathrm{MgCl}_{2}, 4 \mathrm{ATP}$ (Sigma), 0.4 GTP (Sigma) as well as $0.1 \%$ Lucifer yellow; pH was adjusted to 7.2 with $\mathrm{KOH}$. Pyramidal morphology was confirmed with epifluorescence after recording. All experiments were performed in 10 $\mu \mathrm{M}$ bicuculline methiodide (Research Biochemicals, Natick, MA), 10 $\mu \mathrm{M}$ 6-cyano-7-nitroquinoxaline-2,3-dione (CNQX), and $40 \mu \mathrm{M}$ D-2amino-5-phosphonovaleric acid (D-AP-5, Tocris Cookson, Bristol, UK) to block residual background synaptic activity.

To recreate tonic depolarization, long depolarizing current steps were injected into the cell via the recording pipette; current magnitude is reported as $I_{\text {stim }}$. To recreate increased membrane conductance (i.e., shunting), an artificial conductance was applied via dynamic clamp implemented with a Digidata 1200A ADC/DAC board (Molecular Devices) and DYNCLAMP2 software (Pinto et al. 2001) running on a dedicated processor; update rate was $10 \mathrm{kHz}$. For this study, we only report the effects of a single conductance level that was constant at 10 $\mathrm{nS}$ and was associated with a reversal potential of $-70 \mathrm{mV}$; this conductance caused an average $56 \%$ reduction in input resistance. To recreate the voltage noise caused by synaptic input, noisy current (see following text) was injected into the cell, but the effects of noise were not analyzed in this study except for one experiment identified in RESULTS. Recreating the effects of background synaptic activity using dynamic clamp has been previously validated and discussed in detail elsewhere (Chance et al. 2002; Destexhe et al. 2001; Prescott et al. 2006; for review, see Prescott and De Koninck 2008).

Traces were low-passed filtered at $4 \mathrm{kHz}$ and stored on videotape using a digital data recorder (VR-10B, Instrutech; Port Washington, NY). Off-line, recordings were sampled at $10 \mathrm{kHz}$ on a computer using Strathclyde Electrophysiology software (J. Dempster, Department of Physiology and Pharmacology, University of Strathclyde, Glasgow, UK) and analyzed using locally designed software (De Koninck).

\section{Simulations}

Simulations were based on a modified Morris-Lecar model (Morris and Lecar 1981; Rinzel and Ermentrout 1998) that we have analyzed in detail (Prescott et al. 2008). The model is described by the following equations

$$
\begin{gathered}
C \mathrm{~d} V / \mathrm{d} t=I_{\text {stim }}+I_{\text {noise }}-\bar{g}_{\mathrm{Na}} m_{\infty}(V)\left(V-E_{\mathrm{Na}}\right)-\bar{g}_{\mathrm{K}, \mathrm{dr}} w\left(\mathrm{~V}-\mathrm{E}_{\mathrm{K}}\right) \\
-g_{\text {shunt }}\left(V-E_{\text {shunt }}\right)-\bar{g}_{\text {adapt } z} z\left(\mathrm{~V}-\mathrm{E}_{\mathrm{K}}\right) \\
\mathrm{d} w / \mathrm{d} t=\phi_{w} \frac{w_{\infty}(V)-w}{\tau_{w}(V)} \\
\mathrm{d} z / \mathrm{d} t=\left[\frac{1}{\left.1+e^{\left(\beta_{\mathrm{z}}-V\right) / \gamma_{z}}-z\right] / \tau_{z}}\right. \\
m_{\infty}(V)=0.5\left[1+\tanh \left(\frac{V-\beta_{m}}{\gamma_{m}}\right)\right] \\
w_{\infty}(V)=0.5\left[1+\tanh \left(\frac{V-\beta_{w}}{\gamma_{w}}\right)\right] \\
\tau_{w}(V)=1 / \cosh \left(\frac{V-\beta_{w}}{2 \cdot \gamma_{w}}\right)
\end{gathered}
$$

This minimal model was used instead of more complex, higher dimensional models (e.g., Hodgkin-Huxley) because it is amenable to 
dynamical analysis that can be used to explain key features of the model's behavior (see following text). For example, the Morris-Lecar model neglects sodium channel inactivation since it operates on the same time scale as delayed rectifier channel activation (e.g., Kepler et al. 1992). Our model is modified from the original Morris-Lecar model primarily by the addition of adaptation. All parameters were set according past modeling studies (Prescott et al. 2006, 2008) rather than being quantitatively fit to experimental data, notwithstanding the final set of simulations presented in Figs. 8 and 9.

Unless otherwise stated, $E_{\mathrm{Na}}=50 \mathrm{mV}, E_{\mathrm{K}}=-100 \mathrm{mV}, E_{\text {shunt }}=$ $-70 \mathrm{mV}, \bar{g}_{\mathrm{Na}}=20 \mathrm{mS} / \mathrm{cm}^{2}, \bar{g}_{\mathrm{K}, \mathrm{dr}}=20 \mathrm{mS} / \mathrm{cm}^{2}, \phi_{\mathrm{w}}=0.25, C=2$ $\mu \mathrm{F} / \mathrm{cm}^{2}, \beta_{\mathrm{m}}=-1.2 \mathrm{mV}, \gamma_{\mathrm{m}}=18 \mathrm{mV}, \beta_{\mathrm{w}}=-9 \mathrm{mV}$, and $\gamma_{\mathrm{w}}=10$ $\mathrm{mV}$. Low- and high-conductance states were simulated by changing $g_{\text {shunt }}$ from 2 to $4 \mathrm{mS} / \mathrm{cm}^{2}$. In additional simulations not reported here, the $2 \mathrm{mS} / \mathrm{cm}^{2}$ increase in $g_{\text {shunt }}$ was associated with a reversal potential of $-57 \mathrm{mV}$, which more accurately reflects the net reversal potential for background synaptic activity (see following text); results were qualitatively unchanged from simulations in which the reversal potential was $-70 \mathrm{mV}$. Adaptation comprised two separate currents: M-type $\mathrm{K}^{+}$current with $\bar{g}_{\mathrm{M}}=2 \mathrm{mS} / \mathrm{cm}^{2}, \tau_{\mathrm{z}}=200 \mathrm{~ms}, \beta_{\mathrm{z}}=-30$ $\mathrm{mV}$, and $\gamma_{\mathrm{z}}=5 \mathrm{mV}$ and calcium-activated $\mathrm{K}^{+}$current with $\bar{g}_{\mathrm{AHP}}=$ $1 \mathrm{mS} / \mathrm{cm}^{2}, \tau_{\mathrm{z}}=200 \mathrm{~ms}, \beta_{\mathrm{z}}=0 \mathrm{mV}$, and $\gamma_{\mathrm{z}}=5 \mathrm{mV}$. Activation of $I_{\mathrm{M}}$ and $I_{\mathrm{AHP}}$ were controlled separately by $z_{\mathrm{M}}$ and $z_{\mathrm{AHP}}$.

Weak noise was included in all simulations investigating membrane potential oscillations (MPOs) to replicate noise that is still present in real neurons after blockade of fast synaptic input. This noise prevents the model from being unphysiologically stable by perturbing the system from its fixed point; MPOs are generated as the system returns toward its fixed point, meaning at least some noise is necessary for the generation of irregular MPOs like those observed experimentally (Dorval and White 2005; Erchova et al. 2004; Hutcheon and Yarom 2000). Noisy current was modeled as an Ornstein-Uhlenbeck process $\mathrm{d} I_{\text {noise }} / \mathrm{d} t=-I_{\text {noise }} / \tau_{\text {noise }}+\sigma_{\text {noise }} N(0,1)$ where $N$ is a random number with 0 mean and unit variance, and $\sigma_{\text {noise }}=0.1$ or $0.01 \mu \mathrm{A} / \mathrm{cm}^{2}$ when $\tau_{\text {noise }}=5$ or $500 \mathrm{~ms}$, respectively, to give equivalent variance in $I_{\text {noise }}$ which equals $\sigma^{2} \tau / 2$ (see Gillespie 1996). These values of $\sigma_{\text {noise }}$ are small compared with those used to replicate synaptic noise.

For the model, all conductances and currents are reported in densities (i.e., per unit area). For comparison with experiments in which, for example, $I_{\text {stim }}$ is not expressed per unit area, the model neuron can be assumed to have a surface area of about $300 \mu \mathrm{m}^{2}(=3 \times$ $10^{-6} \mathrm{~cm}^{2}$ ). In other words, $I_{\text {stim }}$ in $\mu \mathrm{A} / \mathrm{cm}^{2}$ multiplied by $3 \times 10^{-6}$ $\mathrm{cm}^{2} \times 10^{6} \mathrm{pA} / \mu \mathrm{A}$ gives $I_{\text {stim }}$ in $\mathrm{pA}$.

\section{Dynamical analysis}

Our modified Morris-Lecar model is ideally suited for dynamical analysis techniques because it comprises the minimum number of variables (dimensions) required to reproduce the phenomena of interest. Ideally, the system can be simplified to two dimensions, i.e., the interaction between a fast activation variable (e.g., $V$ ) and a slower recovery variable (e.g., $w$ or $z$ ). That interaction can be visualized by plotting the fast variable against the slower variable to create a phase portrait. Nullclines represent areas in phase space where a given variable remains constant. How the nullclines intersect (i.e., whether the intersection is stable or unstable) determines whether the system evolves toward a fixed point or toward a limit cycle (i.e., subthreshold membrane potential or repetitive spiking, respectively). Stability of the fixed point can be determined by local stability analysis, which involves linearizing the nullclines in the vicinity of their intersection and finding the eigenvalues of the Jacobian matrix. This analysis leads to inequalities discussed in Fig. 5 (see Borisyuk and Rinzel 2005 for full derivations) and helps explain how voltage trajectories evolve toward or away from the fixed point, including the frequency of subthreshold MPOs.

The original Morris-Lecar model is two dimensional (2D). Our model is $3 \mathrm{D}$ because of the addition of slow adaptation. Because adaptation develops slowly relative to the other variables, $z$ (which controls adaptation; see preceding text) can be approximated as constant over some time window, thus allowing the model to be reduced from $3 \mathrm{D}$ to $2 \mathrm{D}$; in other words, $z$ is treated as a parameter and behavior is explained based on dynamical interaction between $V$ and $w$. Initial analysis is based on this assumption. In the final two figures, however, slow changes in $z$ are shown to be important for generating low-frequency oscillations. In this case, the system can still be approximated as 2D but with oscillations being explained by dynamical interaction between $V$ and $z$ (see RESULTS).

To determine the effects of certain parameters, the parameter of interest (e.g., $\left.I_{\text {stim }}\right)$ can be continuously varied to determine its effects on the system's behavior, which will be reflected in the nullcline intersection. Of particular interest are bifurcations or abrupt transitions in the system's behavior, such as the transition between quiescence and repetitive spiking. See Rinzel and Ermentrout (1998) for a more detailed discussion of these methods in the context of neuronal excitability or Strogatz (1998) for an in-depth explanation.

\section{R E S U L T S}

\section{Effects of shunting and adaptation on excitability in a modified Morris-Lecar model}

Class 1 and 2 neurons initiate spikes through distinct dynamical mechanisms. Those dynamics represent competition between inward (depolarizing) and outward (hyperpolarizing) currents active at perithreshold potentials. In class 1 neurons, net current is inward at steady state. Sustained weak inward current allows the neuron to maintain slow repetitive spiking. This is manifested dynamically as a saddle-node on invariant circle (SNIC) bifurcation. In class 2 neurons, on the other hand, net current is outward at steady state. In this case, spike initiation requires that fast-activating inward current produces suprathreshold depolarization before slower-activating outward current becomes strongly activated (i.e., before net current becomes outward). This is manifested dynamically as a Hopf bifurcation. Importantly, class 2 neurons cannot maintain spiking below a critical rate lest slow-activating outward current overtake fast-activating inward current. For more detailed discussion of these points, see Izhikevich (2007) or Prescott et al. (2008).

A relative increase in outward current therefore encourages spike initiation through a Hopf bifurcation (class 2 excitability), whereas a relative increase in inward current encourages spike initiation through a SNIC bifurcation (class 1 excitability). Adaptation is caused by potassium currents the reversal potential of which is around $-90 \mathrm{mV}$ and, in the case of the M-type current $I_{\mathrm{M}}$, clearly contributes an outward current at perithreshold potentials. Similarly, shunting will produce an outward current at perithreshold potentials if its reversal potential is more hyperpolarized than threshold. Chance et al. (2002) calculated the conductance-weighted average of excitatory and inhibitory reversal potentials to be $-57 \mathrm{mV}$, which is consistent with average membrane potentials reported by Destexhe and Paré (1999). Therefore shunting and adaptation both contribute outward current and are therefore predicted to encourage class 2 excitability. Decreased inward current (through cumulative sodium channel inactivation, for example) could cause a similar shift in net current with equivalent consequences for excitability (see following text).

To begin, we tested our theoretical prediction that increased outward current contributed by shunting and/or adaptation 
encourages class 2 excitability. First, we performed experiments in which in vivo-like conditions were replicated in acute brain slices using prolonged depolarizing current injections to activate adaptation with or without dynamic clamp to artificially increase membrane conductance (i.e., cause shunting) (Fig. 1A, top). Then,
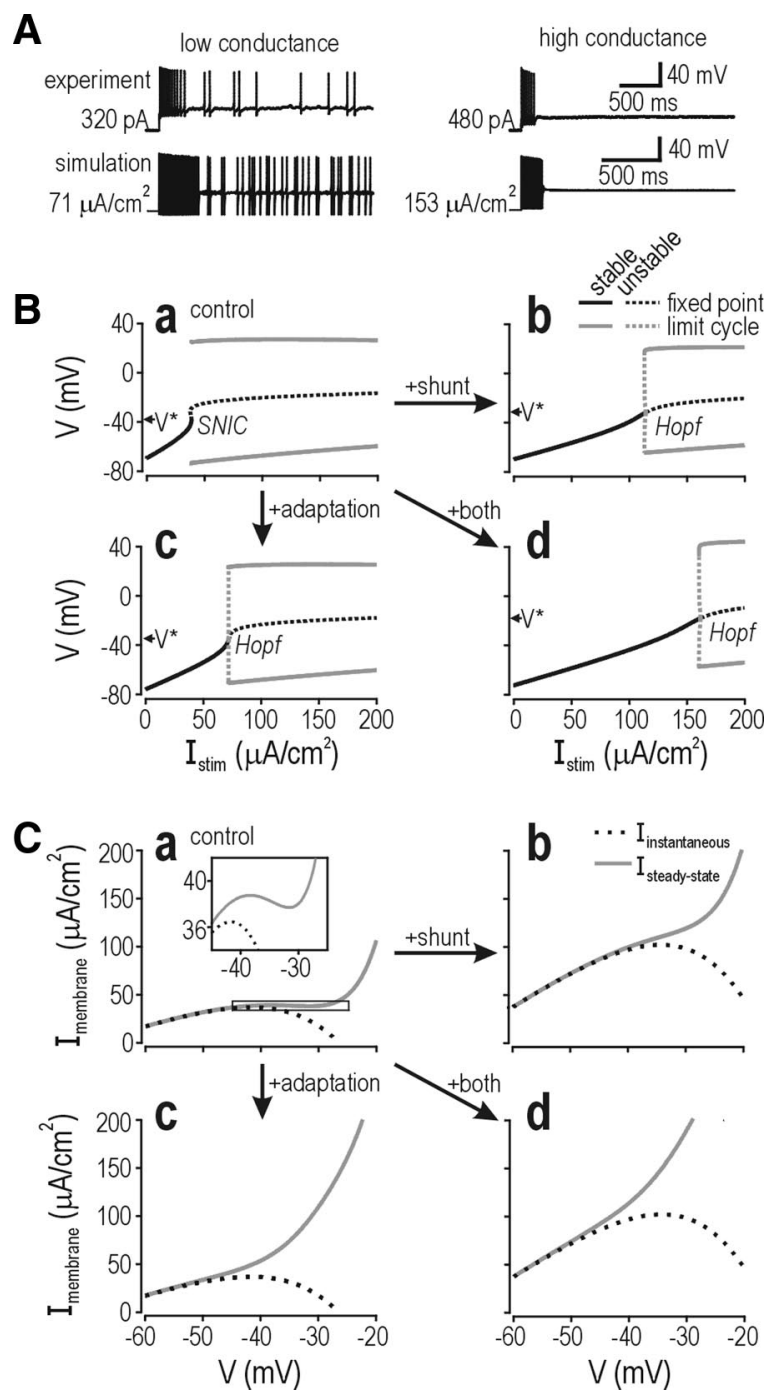

FIG. 1. Shunting and adaptation convert neuronal excitability from class 1 to class 2. A, top: sample responses from a typical CA1 hippocampal pyramidal cell with and without shunting introduced artificially by dynamic clamp. Bottom: response from a modified Morris-Lecar model adjusted to qualitatively reproduce the experimentally observed response pattern. $B$ : bifurcation diagrams were generated by varying stimulus intensity $\left(I_{\text {stim }}\right)$. The bifurcation represents the qualitative change in behavior from quiescence to repetitive spiking. Under control conditions (no adaptation or shunting; $a$ ), the model generated spikes through a saddle-node on invariant circle (SNIC) bifurcation, consistent with class 1 excitability. When shunting $(b)$, adaptation $(c)$, or both (d) were introduced, the model switched to generating spikes through a subcritical Hopf bifurcation, consistent with class 2 excitability. Shunting and adaptation both caused voltage at the bifurcation $\left(V^{*}\right)$ to increase, which reflects the increased outward current contributed by shunting and adaptation at perithreshold potentials (see Fig. 2). For bifurcation analysis, activation of $I_{\mathrm{M}}(0<z<1)$ was held constant at the steady-state value of $z$ measured from responses in A. $C: I-V$ curves based on conditions in $B$. The steady-state $I-V$ curve was nonmonotonic (i.e., it had a region of negative slope; see inset) under control conditions $(a)$, whereas it was monotonic after adaptation and/or shunting were added $(b-d)$. Instantaneous $I-V$ curve was calculated as $I_{\mathrm{Na}}+$ $I_{\text {leak }}$ while steady-state $I$ - $V$ curve was calculated as $I_{\mathrm{Na}}+I_{\text {leak }}+I_{\mathrm{K}, \mathrm{dr}}\left(+I_{\mathrm{M}}\right.$ if present). starting with a modified Morris-Lecar model the parameters of which were set to values that give class 1 excitability, we added shunting and adaptation to reproduce the experimentally observed prohibition of repetitive spiking in the high-conductance state (Fig. 1A, bottom); the basis for this phenomenon has already been described in detail (Prescott et al. 2006) and results from nonlinearly increased activation of $I_{\mathrm{M}}$ at perithreshold potentials in the high-conductance state.

With model parameters thus established, we used bifurcation analysis to ascertain the spike-initiating mechanism under different conditions. Under control conditions (i.e., without shunting or adaptation), spikes were generated through a SNIC bifurcation (Fig. $1 \mathrm{Ba}$ ), which is indicative of class 1 excitability. Consistent with the predicted effects of an increase in outward current (see preceding text), the spike-initiating mechanism switched to a subcritical Hopf bifurcation when shunting and/or adaptation were included in the model (Fig. $1 B, b-d$ ), thus demonstrating conversion to class 2 excitability. The switch in bifurcation mechanism was paralleled by a change in the steady-state $I-V$ curve, from nonmonotonic under control conditions (Fig. $1 \mathrm{Ca}$ ) to monotonic when shunting and/or adaptation were added (Fig. $1 C, b-d$ ). A nonmonotonic curve means there is a local maximum above which depolarization activates net inward current at steady state-this is the biophysical basis for spike initiation through a SNIC bifurcation. A monotonic curve has no local maximum, meaning fastactivating inward current must cause suprathreshold depolarization before slower-activating outward current causes net steady-state current to become outward-this is the biophysical basis for spike initiation through a Hopf bifurcation (see preceding text). The change in shape of the steady-state $I-V$ curve is therefore consistent with the relative increase in outward current contributed by shunting and/or adaptation which causes net current at perithreshold potentials to change direction (see following text).

\section{Effects of increased outward current on voltage threshold}

Shunting and adaptation both caused a depolarizing shift in spike threshold $\left(V^{*}\right)$ according to bifurcation analysis in Fig. $1 B$; threshold can be ascertained from voltage at the bifurcation. The shift is explained by the fact that inward current mediated by the fast sodium current $I_{\mathrm{Na}}$ must offset increased outward current contributed by shunting and adaptation; increased depolarization is required to activate $I_{\mathrm{Na}}$ more strongly to provide this increased inward current (Fig. $2 A$ ) and contributes to the depolarizing shift in $V^{*}$ (Prescott et al. 2006). Importantly, the shift in $V^{*}$ occurs in a steep region of the voltage-dependent activation curve for the delayed rectifier potassium current $I_{\mathrm{K}, \mathrm{dr}}$ (Fig. $2 B$ ) and for $I_{\mathrm{M}}$ (see following text). Consequently, a small change in $V^{*}$ allows dramatically increased activation of potassium currents at perithreshold potentials: this compounds the increase in outward current, exacerbates the depolarizing shift in $V^{*}$, and causes the steadystate $I-V$ curve to become monotonic (Fig. 2C).

Simulations therefore predicted that shunting and adaptation cause an increase in $V^{*}$. We therefore measured $V^{*}$ in experimental data based on the voltage at which $\mathrm{d}^{2} V / \mathrm{d} t^{2}$ exceeded a cutoff value indicative of a spike. As predicted, both shunting and adaptation caused a depolarizing shift in $V^{*}$ (Fig. 3, $A$ and $B)$. Furthermore, the experimentally measured shift in $V^{*}$ 

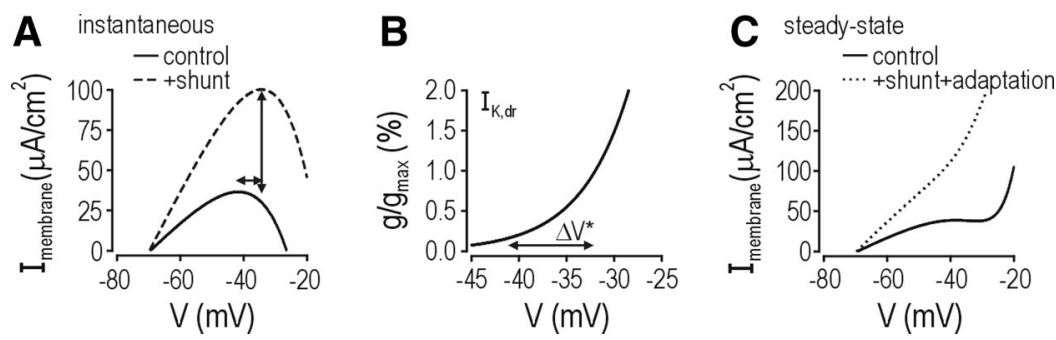

FIG. 2. Basis for and consequences of a depolarizing shift in voltage threshold $\left(V^{*}\right)$. A: height of the local maximum of the instantaneous $I$ - $V$ curve was increased by the outward current contributed by shunting $(\uparrow)$. It was also shifted rightward $(\leftrightarrow)$ because of the increased depolarization required to more strongly activate fast sodium current $I_{\mathrm{Na}}$ to counterbalance the increase in outward current; this contributes to the depolarizing shift in $V^{*}$ and also allows for stronger subthreshold activation of the delayed rectifier potassium current $I_{\mathrm{K}, \mathrm{dr}}($ see $B)$ and the M-type potassium current $I_{\mathrm{M}}$. $B: I_{\mathrm{K}, \mathrm{dr}}$ was only weakly activated at $V^{*}$ under control conditions; however, because $V^{*}$ sits in steep regions of its activation curve, modest changes in $V^{*}$ (like in Fig. 1) allow for dramatically increased perithreshold activation of $I_{\mathrm{K}, \mathrm{dr}} \cdot \leftrightarrow$, range of $V^{*}$ identified in Fig. 1B.C: increased perithreshold activation of potassium currents causes net current to become outward at steady state, hence the "unbending" of the steady-state $I$ - $V$ curve, i.e., conversion from nonmonotonic to monotonic, as $V^{*}$ is shifted.

occurred in a steep region of the activation curve for $I_{\mathrm{K}, \mathrm{dr}}$, assuming typical parameters used to model Hodgkin-Huxley channels in hippocampal pyramidal neurons (Fig. 3C), and would therefore allow nonlinearly increased activation of $I_{\mathrm{K}, \mathrm{dr}}$, consistent with the Morris-Lecar model reported in Fig. $2 B$. These data are therefore consistent with the postulated shift in balance of perithreshold currents that would account for the switch from class 1 to class 2 excitability.

\section{Effects of shunting and adaptation on shape of the f-I curve}

We then sought more direct evidence for a functional switch in excitability caused by shunting and adaptation. Because Hodgkin's original basis for classification was the shape of the $f-I$ curve (see INTRODUCTION), we tested whether shunting and adaptation changed the $f-I$ curve from continuous to discontinuous, consistent with a switch in excitability from class 1 to class 2.

Shunting had two effects on the $f-I$ curve according to simulations: it shifted the curve rightward and increased the minimum sustainable firing rate $\left(f_{\min }\right)$ to $>0$ spike/s, thus making the curve discontinuous (Fig. $4 A$ ). To determine effects of shunting on $f_{\min }$ in experimental data while minimizing the
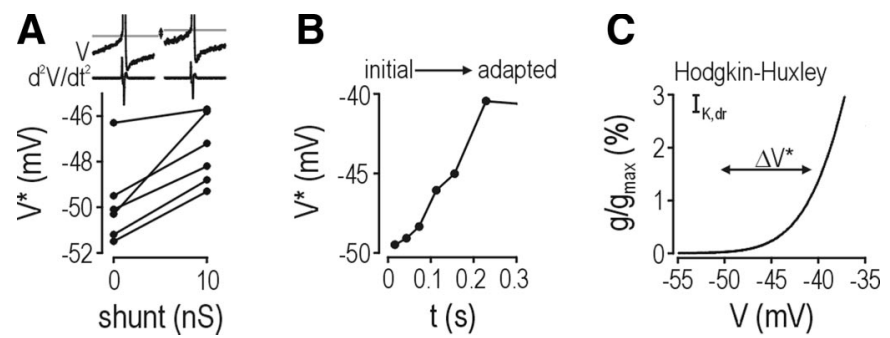

FIG. 3. Experimental evidence for a depolarizing shift in voltage threshold $\left(V^{*}\right)$. A: a 10 -nS shunt produced a significant $2.3 \pm 0.5$ (SE) $\mathrm{mV}$ depolarizing shift in $V^{*}(P<0.01$, paired $t$-test, $n=6$ cells $) . V^{*}$ was estimated as the voltage at which $\mathrm{d}^{2} V / \mathrm{d} t^{2}$ (which corresponds to rate of activation of $I_{\text {membrane }}$ ) exceeded a cutoff value $5 \times$ the root-mean-square noise in the baseline $\mathrm{d}^{2} V / \mathrm{d} t^{2}$ trace. Based on responses to $I_{\text {stim }} 40 \mathrm{pA}$ greater than rheobase, we measured $V^{*}$ from the 2nd spike within each spike train because the 2 nd spike occurs before adaptation develops, and its analysis is not confounded by initial membrane charging, as can occur with the 1st spike (modified from Prescott et al. 2006). $B$ : adaptation also increased $V^{*}$ measured, as in $C$, for sequential spikes within the initial burst. Data here are from a typical response and show a dramatic increase in $V^{*}$ as adaptation develops. $C$ : experimentally measured shifts in $V^{*}$ $(\leftrightarrow)$ sit within the steep region of the activation curve for $I_{\mathrm{K}, \mathrm{dr}}$ according to a typical Hodgkin-Huxley model used for hippocampal pyramidal cells (Traub and Miles 1991). influence of adaptation, we measured instantaneous firing rate from the reciprocal of the first interspike interval or ISI and compared $f$-I curves in the low- and high-conductance states. Like in simulations, shunting shifted experimental $f$-I curves rightward and increased $f_{\min }$ (Fig. $4 B$ ). Unlike in simulations, it is impossible to demonstrate experimentally that the neuron can spike at rates arbitrarily close to 0 spike/s (given the residual noise from ion channels, etc.), but, across all neurons tested, $f_{\min }$ increased significantly from $3.5 \pm 1.0$ spike/s under control conditions to $16.4 \pm 1.4$ spike/s when a $10-n S$ shunt was applied to the neuron ( $P<0.001, t$-test, $n=6$ cells; Fig. $4 C$ ).

To determine if adaptation caused a similar increase in $f_{\min }$, we measured instantaneous firing rate from the reciprocal of the last ISI within the initial burst (i.e., after development of adaptation). Once adapted, the same cell illustrated in Fig. 4B could not sustain spiking below $\sim 20$ spike/s regardless of $I_{\text {stim }}$ (Fig. 4D). Across all neurons, adaptation significantly increased $f_{\min }$ in both the low- and high-conductance states to $16.2 \pm 1.6$ and $25.5 \pm 1.6 \mathrm{spike} / \mathrm{s}$, respectively $(P<0.005$; Fig. 4E).

Thus shunting and tonic depolarization (which elicits adaptation) comparable to that experienced by neurons in vivo caused neuronal $f$ - $I$ curves to become discontinuous, consistent with the neuron switching from class 1 to class 2 excitability. By causing a switch to class 2 excitability, we predicted that shunting and adaptation encourage neurons to oscillate or resonate (i.e., respond more strongly to certain input frequencies), whereas those same neurons would not oscillate under control conditions when they are class 1 excitable.

\section{Effects of shunting and adaptation on MPOs}

We begin here by explaining the dynamical basis for the preceding prediction, i.e., why class 2 neurons oscillate whereas class 1 neurons do not. Class 1 neurons initiate their spikes on the basis of subthreshold inward current causing a SNIC bifurcation. This is reflected in the nonmonotonic steady-state $I-V$ curve (Fig. $1 C a$ ) and can be summarized by local stability analysis at the fixed point (see METHODS), which shows that $\partial I_{\mathrm{ss}} / \partial V=0$ at the saddle-node (Fig. 5Aa). The implications are that fast-activating inward current $\left(I_{\mathrm{Na}}\right)$ does not compete with slow-activating outward current $\left(I_{\mathrm{K}, \mathrm{dr}}\right.$ or $\left.I_{\mathrm{M}}\right)$ because the latter only starts to activate at suprathreshold voltages - this is why steady-state current is inward at perithreshold potentials and why the corresponding $I-V$ curve has 

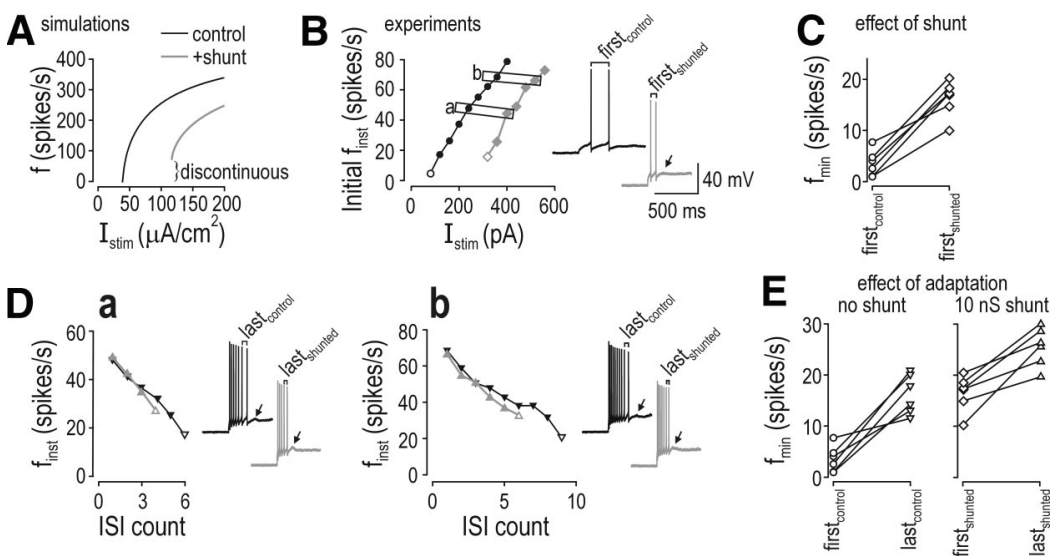

FIG. 4. Shape of the $f-I$ curve depends on stimulus conditions. A: $F-I$ curves from model without adaptation. Shunting shifted the curve rightward and made it discontinuous, i.e., the shunted neuron could not sustain spiking below a minimum rate $\left(f_{\min }\right)$ of $\sim 80$ spike/s. A continuous $f-I$ curve is consistent with class 1 excitability, whereas a discontinuous one is consistent with class 2 excitability. B: F-I curves from CA1 pyramidal neurons exhibited the same effects of shunting. Instantaneous firing rate $\left(f_{\text {inst }}\right)$ was measured from the reciprocal of interspike intervals (ISIs); initial $f_{\text {inst }}$ was calculated from the first ISI, which is relatively unaffected by slow adaptation. Sample responses highlight the shunting-induced increase in $f_{\min }$, which was determined as initial $f_{\text {inst }}$ for the minimum stimulus intensity eliciting $\geq 2$ spikes $\left(I_{\min 2}\right)$. Rectangles labeled $a$ and $b$ indicate responses shown in $D$. Arrow here and in $D$ point to the subthreshold oscillation that follows the last spike of the initial burst; presence of an oscillation is consistent with class 2 excitability, whereas its absence is consistent with class 1 excitability (see Fig. 7). $C$ : based on responses to $I_{\min 2}$, shunting caused a significant increase in $f_{\min }(P<0.001$, paired $t$-test, $n=6$ cells). $D$ : sample responses from same cell as in $B$ show adaptation-induced increase in $f_{\min }$. Instantaneous firing rate was measured from successive ISIs. $I_{\text {stim }}$ was adjusted to give the same initial firing rate in the low- and high-conductance states (like in Fig. 1A); each graph shows different initial firing rates as indicated in $B$. Regardless of initial firing rate (i.e., $a$ and $b$ show equivalent results), $f_{\min }$ calculated from the last ISI of the burst was higher than $f_{\min }$ calculated from the first ISI elicited by $I_{\min 2}$ (compare with $B$ ). E: adaptation caused a significant increase in $f_{\min }$ according to comparison of $f_{\min }$ calculated from the last ISI (elicited by $\left.80 \mathrm{pA}>I_{\min 2}\right)$ with $f_{\min }$ calculated from the 1 st ISI (elicited by $\left.I_{\min 2}\right)$; that was true in both the low- and high-conductance states $(P<0.005$, paired $t$-test, $n=6$ cells).

a region of negative slope. In class 2 neurons, on the other hand, spike initiation occurs through a Hopf bifurcation because net current is outward at threshold, as reflected in the monotonic steady-state $I-V$ curve (Fig. $1 C, b-d$ ). This means that $I_{\mathrm{Na}}$ must compete with $I_{\mathrm{K}, \mathrm{dr}}\left(\right.$ or $\left.I_{\mathrm{M}}\right)$ in a time-dependent manner. Subthreshold MPOs occur when inward current starts to activate but outward current catches up before a full spike occurs; this trajectory is manifested on the phase plane as spiraling around a stable focus $\left(-\frac{1}{C} \frac{\partial I_{\text {inst }}}{\partial V}<\frac{\phi_{w}}{\tau_{w}}\right.$ at the fixed point; Fig. 5A, $b$ and $c$ ) (see also Hutcheon and Yarom 2000). Repetitive spiking occurs when that focus becomes unstable via a subcritical Hopf bifurcation $\left(-\frac{1}{C} \frac{\partial I_{\text {inst }}}{\partial V}>\frac{\phi_{w}}{\tau_{w}}\right)$.

By relating the generation of MPOs to the nullcline intersection, we can predict how conditions impact MPO frequency. MPO frequency reflects the rate of spiraling around the focus, which is reflected in the value of the complex component of the eigenvalue found by local stability analysis (Kaplan and Glass 1995). Equivalently, MPO frequency can be estimated from the phase plane: oscillations cannot occur when the $V$ - and $w$ nullclines intersect tangentially, but MPO frequency increases as the nullcline intersection becomes less acute (i.e., as the angle becomes less sharp) (Fig. 5A, middle row). The preceding is true for a given set of rate constants, but MPO frequency also depends on how rapidly outward current activates (controlled by $\phi_{\mathrm{w}}$ or $\tau_{\mathrm{w}}$ ) relative to instantaneous activation of inward current. Importantly, MPO frequency and $f_{\min }$ are directly related insofar as MPO frequency is less than $f_{\min }+\Delta f$ (Fig. 5A, bottom row), where $\Delta f$ accounts for subthreshold MPOs occurring in the bistable region of the bifurcation diagram (Fig. 5B).

Thus based on phase plane geometry, we know that class 2 neurons should oscillate whereas class 1 neurons should not.
Indeed the model did not exhibit subthreshold MPOs when depolarized to within a few millivolts of threshold under control conditions, when excitability was class 1 (Fig. 6A, black), but subthreshold MPOs were observed after the model was converted to class 2 excitability (Fig. 6A, gray). To show that MPOs resulted from the switch in excitability rather than being a specific consequence of shunting or adaptation, the model shown in Fig. $6 A$ was converted to class 2 excitability by changing an unrelated parameter, $\beta_{\mathrm{m}}$, which caused a depolarizing shift in the voltage-dependent activation curve for $I_{\mathrm{Na}}$. Because greater depolarization was required to activate $I_{\mathrm{Na}}$, this parameter change caused a depolarizing shift in $V^{*}$ (like in Fig. 2A); as a result, spikes were generated through a Hopf bifurcation (bifurcation diagram not shown). Reducing the maximal sodium conductance $\left(\bar{g}_{\mathrm{Na}}\right)$ has a comparable effect (see following text).

If, instead of adjusting $\beta_{\mathrm{m}}$, we added adaptation or shunting to the control model, the model exhibited subthreshold MPOs as predicted by its conversion to class 2 excitability (Fig. 6B). Furthermore, as predicted by local stability analysis (see corresponding phase planes in Fig. 5), the shunted neuron exhibited higher frequency MPOs than the adapted neuron given the parameters used (Fig. 6B). Also the amplitude (power) of MPOs was substantially less in the shunted neuron because low input resistance in the shunted neuron mitigated voltage changes. By stimulating our model with periodic inputs with different frequencies, we confirmed that class 2 models resonated at frequencies near their oscillation frequency, whereas the class 1 (control) model did not resonate, which is consistent with its inability to oscillate (Fig. 6C); both results are consistent with previous work (e.g., Izhikevich 2007).

We then compared these results with experimental data. Our recordings revealed subthreshold MPOs when CA1 pyramidal 

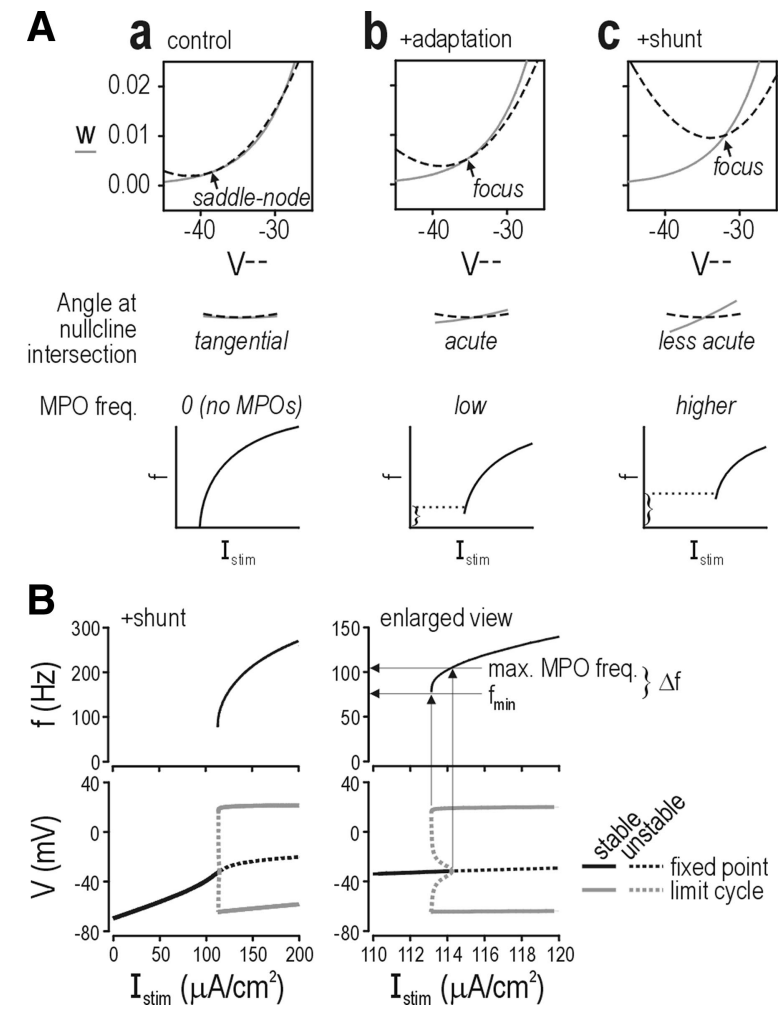

FIG. 5. The dynamical basis for subthreshold membrane potential oscillations (MPOs). A: spike-initiating dynamics can be visualized on the phase plane in which model variables $V$ and $w$ are plotted relative to one another; nullclines represent everywhere in phase space where $V$ or $w$ do not change and are shown here for conditions at the bifurcation. In control $(a)$, a spike cannot be generated until 2 fixed points (a saddle and a node) come together and are destroyed through a SNIC bifurcation, which occurs when the $w$ - and $V$ nullclines meet tangentially. $\partial I_{\mathrm{ss}} / \partial V=0$ at the fixed point, which means net current is 0 ; in other words, spiking begins in a class 1 neuron when net current switches direction from outward to inward. With adaptation $(b)$ or shunting (c) or both (not shown), the nullclines intersect at a single fixed point that switches from stable to unstable at the moment of the Hopf bifurcation. $-\frac{1}{C} \frac{\partial I_{\text {inst }}}{\partial V}=\frac{\phi_{w}}{\tau_{w}}$ at the fixed point, which means fast-activating inward current balances slower-activating outward current; in other words, spiking begins when activation of $I_{\mathrm{K}, \mathrm{dr}}$ cannot keep up with activation of $I_{\mathrm{Na}}$. Nonetheless, when $-\frac{1}{C} \frac{\partial I_{\text {inst }}}{\partial V}<\frac{\phi_{w}}{\tau_{w}}$, noisy voltage fluctuations can cause $I_{\mathrm{Na}}$ to begin to activate before $I_{\mathrm{K}, \mathrm{dr}}$ catches up, which results in a depolarizing/hyperpolarizing sequence manifested as a subthreshold MPO. This is evident on the phase plane as spiralling around the fixed point or focus (not shown). Rate of spiralling (i.e., frequency of oscillations) can be inferred from how acutely the nullclines intersect and are less than $f_{\min }+\Delta f$ (see following text). $B$ : the bifurcation diagram (bottom left) and $f-I$ curve (top left) are shown for conditions in Ac. Right: enlarged view of left panels. Notice the region of bistability, i.e., where the fixed point and limit cycle overlap. Because of this bistability, subthreshold MPOs can have a maximum frequency that is slightly higher than $f_{\min }$ (as indicated $\rightarrow$ ); we refer to this difference as $\Delta f$.

neurons were tonically depolarized. Oscillations were not necessarily obvious in the raw data, but power spectra showed a clear peak in the $\theta$ frequency range (Fig. 7A). In the lowconductance state, frequency at the peak of the power spectrum corresponded to the interval between doublet spikes in the sample traces. In the high-conductance state, amplitude of MPOs was reduced (i.e., power was reduced and spiking was virtually abolished), but there was still a clear peak in the power spectrum, which was shifted to a higher frequency than in the low-conductance state (Fig. 7A). Changes in MPO frequency and amplitude were consistent with the effects of shunting observed in simulations (see Fig. 6B). Similarly, MPO frequency increased as $I_{\text {stim }}$ was increased (Fig. 7B), consistent with stronger stimulation eliciting stronger adaptation (i.e., larger outward current), which is again consistent with the model (simulation data not shown). As in our model, we confirmed that oscillating CA1 pyramidal neurons also resonated at $\theta$ frequency when stimulated with periodic input (Fig. 7C).

To determine whether CA1 pyramidal neurons could resonate without being tonically depolarized, we stimulated them with noisy current containing frequencies spanning the $\theta$ range (Fig. 7D, inset). This stimulation causes intermittent near-threshold depolarizations and can be used to reveal resonance the same way as periodic input (Schreiber et al. 2004). Using this protocol, we determined that CA1 pyramidal neurons in the low-conductance state that were not tonically depolarized did not resonate (Fig. 7D, black). However, when the same neuron was shunted, power was reduced at low frequencies and increased at high frequencies compared with the unshunted condition, which is consistent with the change in passive membrane properties (i.e., reduced input resistance and membrane time constant) but, more
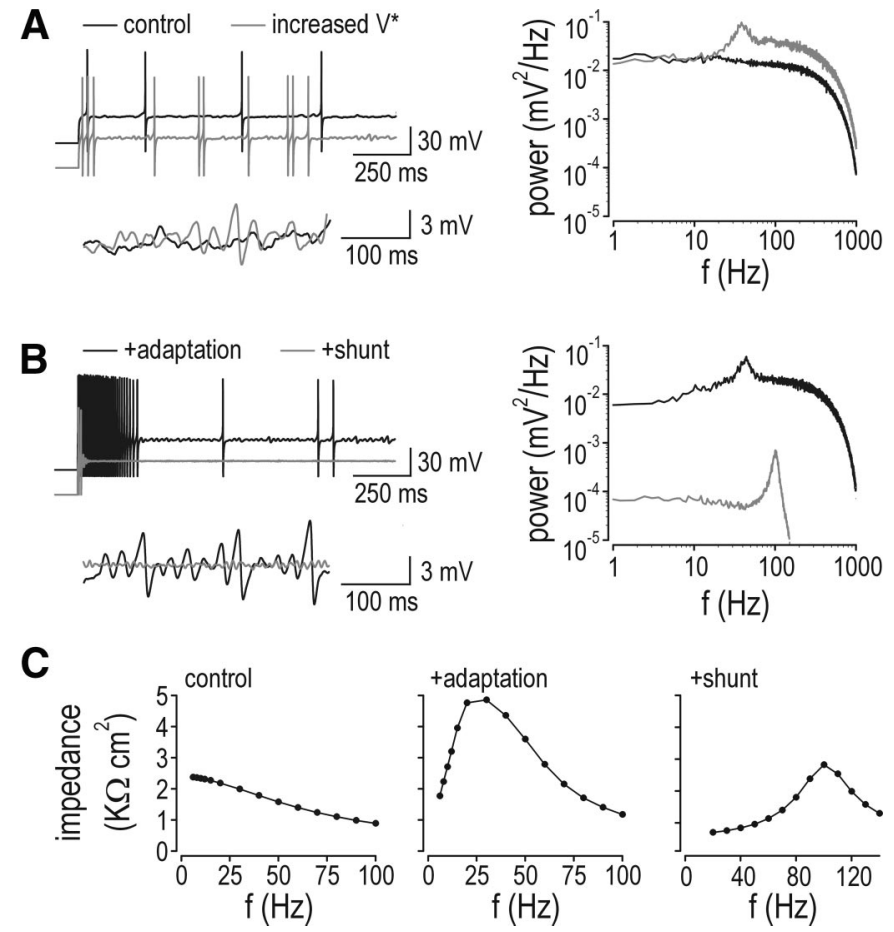

FIG. 6. Occurrence and frequency of subthreshold MPOs in the model. $A$ : just-subthreshold depolarization in the control model (black) did not elicit subthreshold MPOs, consistent with the inability of class 1 neurons to resonate. To demonstrate that resonance depends on excitability and is not a specific consequence of adaptation or shunting, we converted the model to class 2 excitability by changing $\beta_{\mathrm{m}}$ from -1.2 to $0 \mathrm{mV}$, which caused a depolarizing shift in $V^{*}$ via changes in the activation curve for $I_{\mathrm{Na}}$. This new model (gray) clearly exhibited MPOs. For simulations here and in $B$, low-amplitude noise was added as described in METHODS. $B$ : as predicted by their effects on excitability, adding adaptation (black) or shunting (gray) to the control model caused it to oscillate. MPOs were faster in the shunted neuron than in the adapted neuron consistent with our explanation in Fig. 5. In both $A$ and $B$, bottom traces show enlarged view of top traces. $C$ : resonance was also tested by measuring impedance from responses to sine wave stimulation at different frequencies. As expected, the model neuron resonated at frequencies near its oscillation frequency shown in $B$. 

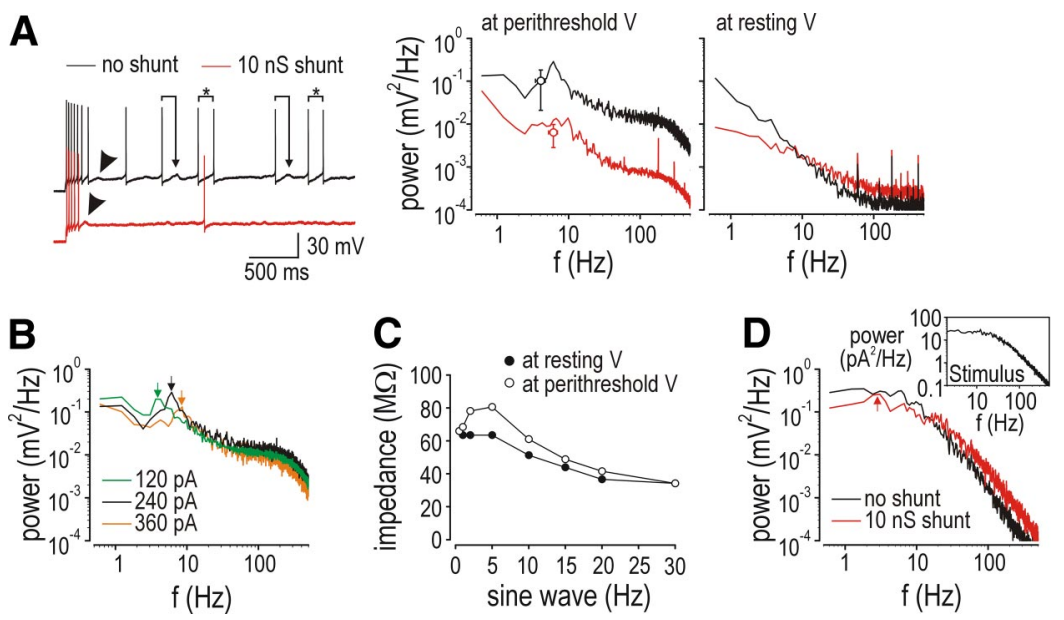

FIG. 7. Occurrence and frequency of subthreshold MPOs in CA1 pyramidal neurons. A: traces show a typical response from a pyramidal neuron in the lowand high-conductance state. In both cases, like in Fig. $4, B$ and $D$, the initial burst was followed by a clear subthreshold MPO $(\swarrow)$. Without shunting, the neuron occasionally fired doublet spikes (*) at an ISI related to the period of subthreshold MPOs $(\rightarrow)$. Power spectra of perithreshold response (left) showed peaks in the $\theta$ frequency range; that peak shifted to a slightly higher frequency when the neuron was shunted, consistent with the effects of increased outward current (compare with Fig. $6 B$ ). $\circ$, mean oscillation frequency and power $( \pm S D)$ based on power spectral analysis of the 6 neurons analyzed. Oscillation frequency was significantly increased while power was significantly decreased by shunting $(P<0.01$ and $P<0.05$, respectively; paired $t$-test). Power spectra on right show lack of MPOs when neuron was not tonically depolarized. $B$ : oscillation frequency also shifted as $I_{\text {stim }}$ was increased, which is again consistent with the increased outward current caused by increased adaptation (see Fig. 5). C: resonance was also tested by measuring impedance from responses to sine wave stimulation at different frequencies. As expected, all neurons $(n=3)$ resonated at $\theta$ frequency when tonically depolarization $(0)$, but not when resting at $-70 \mathrm{mV}(\bullet)$. $D$ : for these power spectra, the neuron was stimulated with large amplitude noise $\left(\sigma_{\text {noise }}=40 \mathrm{pA}\right)$ that caused intermittent near-threshold depolarizations. The neuron resonated in the $\theta$ frequency range when in the high-conductance state (red) but not when in the low-conductance state (black). All neurons tested with this protocol $(n=3)$ exhibited a peak in their power spectrum between 4 and $6 \mathrm{~Hz}$ when shunted, whereas none resonated in the low-conductance state. These results confirm that class 2 neurons resonate at approximately the same frequency at which they oscillate in low-noise conditions, consistent with previous work (Erchova et al. 2004; Lampl and Yarom 1997). Inset: power spectrum of stimulus.

important here, the neuron exhibited resonance in the $\theta$ frequency range (Fig. $7 D$, red). Experimental data are therefore consistent with shunting and/or tonic depolarization converting CA1 pyramidal neurons from integrators to resonators.

\section{Frequency of membrane potential oscillations}

CA1 pyramidal neurons clearly oscillated in the $\theta$ frequency range (Fig. 7); however, our model neuron oscillated at significantly higher frequencies (Fig. 6). We therefore asked whether our model could produce lower frequency MPOs comparable to those observed experimentally. By reducing $\phi_{\mathrm{w}}$ (which controls how rapidly $g_{\mathrm{K}, \mathrm{dr}}$ activates; see $E q$. 2), MPO frequency was shifted down to the $\theta$ frequency range (Fig. 8A). However, reducing $\phi_{\mathrm{w}}$ enough to produce $\theta$ frequency MPOs caused spikes to become unphysiologically wide (Fig. 8A, inset). This suggests that the process controlling $\theta$ frequency MPOs is significantly slower than the process controlling spike repolarization, and that the model must therefore include a third time scale. As alluded to in METHODS, $I_{\mathrm{M}}$ operates on a slower time scale than $I_{\mathrm{K}, \mathrm{dr}}$; hence, we attempted to generate $\theta$ frequency MPOs in our 3D model by adjusting the parameters controlling $I_{\mathrm{M}}$, most notably $\tau_{\mathrm{z}}$. As predicted, appropriately tuned $I_{\mathrm{M}}$ parameters resulted in $\theta$ frequency MPOs (Fig. $8 B$ ).

Time series in Fig. $8 C$ illustrate how MPOs arise from the competition between inward and outward currents. During high-frequency MPOs, initial depolarization (which causes instantaneous activation of $g_{\mathrm{Na}}$ ) is followed after a short delay by activation of $\mathrm{g}_{\mathrm{K}, \mathrm{dr}}$, which causes hyperpolarization (Fig. $8 \mathrm{Ca}$ ); although tonically activated, $g_{\mathrm{M}}$ does not vary on the time scale of these fast oscillations - this was the basis for approximating $I_{\mathrm{M}}$ as a constant for earlier analysis (see METHODS). During low-frequency MPOs, on the other hand, initial slow depolarization is followed with virtually no delay by activation of $\mathrm{g}_{\mathrm{K}, \mathrm{dr}}$, which means $\mathrm{g}_{\mathrm{K}, \mathrm{dr}}$ does not provide the delayed outward current responsible for generating the oscillatory voltage change; instead, initial depolarization is followed by delayed activation of $g_{\mathrm{M}}$. In other words, slow voltage change allows enough time for $g_{\mathrm{M}}$ to vary, despite its slow kinetics, and thus to participate directly in generating MPOs. This has an important technical implication insofar as $I_{\mathrm{M}}$ cannot be approximated as remaining constant when explaining $\theta$ frequency MPOs. Nonetheless, generation of $\theta$ frequency MPOs can still be treated as a 2D problem (rather than a 3D one) because during slow oscillations, $g_{\mathrm{Na}}$ activates slowly and with kinetics similar to $g_{\mathrm{K}, \mathrm{dr}}$ (see Fig. $8 C b$ ); accordingly, $I_{\mathrm{K}, \mathrm{dr}}$ can be approximated as an instantaneously varying offset in the competition between slow-activating $I_{\mathrm{Na}}$ and ultraslow-activating $I_{\mathrm{M}}$.

The dynamics described above are also evident from phase plane analysis. Recall that $w$ and $z$ control activation of $I_{\mathrm{K}, \mathrm{dr}}$ and $I_{\mathrm{M}}$, respectively. In the $2 \mathrm{D}$ model without $I_{\mathrm{M}}$, shunting converted the intersection between the $V$ - and $w$-nullclines from a saddle-node to a focus (Fig. $8 D a$ ), which is consistent with increased perithreshold activation of $I_{\mathrm{K}, \mathrm{dr}}$ (see Fig. 2); high-frequency MPOs result from spiraling around that focus (see Fig. 5). In the 3D model with $I_{\mathrm{M}}$, the $V$ - and $w$-nullclines intersected at a saddle-node despite adaptation (hence, no high-frequency MPOs), but the $V$ - and $z$-nullclines intersected at a focus (Fig. 8Db), which is consistent with perithreshold activation of $I_{\mathrm{M}} ; \theta$ frequency MPOs result from spiraling around this focus. Spiraling is slower on the $V-z$ phase plane because of $z$ 's long time constant (see preceding text). These changes in phase plane geometry are paralleled by changes in the 

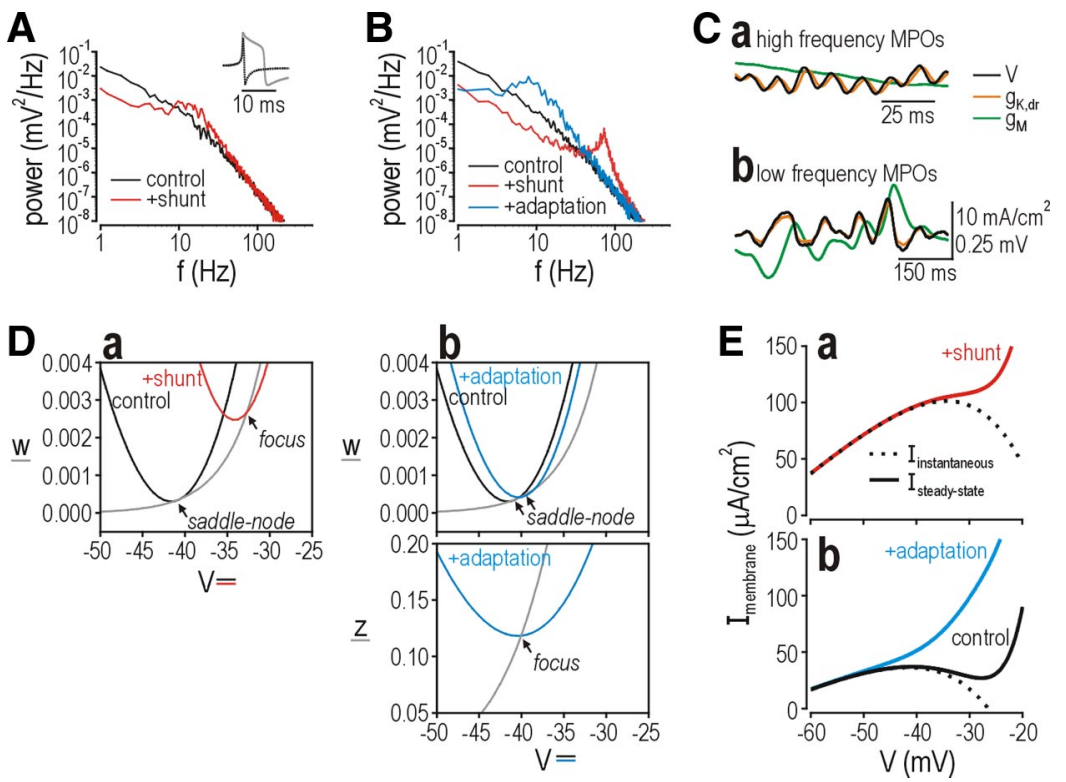

FIG. 8. Oscillation frequency in the model neuron. $A$ : power spectra from modified model in which $\phi_{\mathrm{w}}$ was reduced to 0.008 , thus causing $w$ to change more slowly relative to $V$ (see $E q$. 2). Under these conditions, the model neuron oscillated near $10 \mathrm{~Hz}$ when shunted (rather than near $100 \mathrm{~Hz}$; see Fig. $6 B$ ), whereas the control neuron did not oscillate. However, reducing $\phi_{\mathrm{w}}$ to 0.008 caused spikes to become unnaturally wide; inset: spike when $\phi_{\mathrm{w}}=0.008$ (black) for comparison with spike when $\phi_{\mathrm{w}}=0.25$ (gray). Other parameters were unchanged except $\gamma_{\mathrm{w}}=8 \mathrm{mV}, \bar{g}_{\mathrm{K}, \mathrm{dr}}=30 \mathrm{mS} / \mathrm{cm}^{2}, \tau_{\text {noise }}=500 \mathrm{~ms}$, and $\sigma_{\text {noise }}=0.01 \mu \mathrm{A} / \mathrm{cm}^{2}$. $B$ : alternatively, $\theta$ frequency oscillations could be generated by tuning the time constant for $I_{\mathrm{M}}\left(\tau_{\mathrm{z}}\right)$ to $400 \mathrm{~ms}$. Other parameters were the same as in $A$ except $\phi_{\mathrm{w}}$ was returned to 0.25 . In this model, adaptation caused $\sim 8 \mathrm{-Hz}$ oscillations, whereas shunting continued to produce $\sim 70-\mathrm{Hz}$ oscillations. $C$ : sample responses show the relative timing of changes in voltage (black), $g_{\mathrm{K}, \mathrm{dr}}$ (orange), and $g_{\mathrm{M}}$ (green). Recall that $g_{\mathrm{Na}}$ changes instantaneously with $V$. During high-frequency MPOs $(a)$, activation of $g_{\mathrm{K}, \mathrm{dr}}$ lagged behind changes in $V$, while $g_{\mathrm{M}}$ remained relatively constant. During low-frequency MPOs $(b)$, activation of $g_{\mathrm{M}}$ lagged behind changes in $V$, whereas $g_{\mathrm{K}, \mathrm{dr}}$ kept up (i.e., activated instantaneously) with slow voltage changes. $D$ : phase planes corresponding to conditions in $B$ with nullclines shown for $I_{\text {stim }}$ at the bifurcation. In the 2-dimensional (2D) model without $I_{\mathrm{M}}(a)$, the shunting-induced switch from class 1 to class 2 excitability was evident on the $V$ - $w$ phase plane based on the change in fixed point from saddle-node to focus. The model with adaptation $(b)$ is $3 \mathrm{D}$, and while the fixed point on the $V$-w plane remained a saddle-node, the fixed point on the $V$ - $z$ plane was a focus. In $b, \theta$ frequency oscillations arose from the interaction between $I_{\mathrm{Na}}$ and ultraslow-activating $I_{\mathrm{M}}$ (visualized on the $V$ - $z$ plane) rather than through interaction between $I_{\mathrm{Na}}$ and slow-activating $I_{\mathrm{K}, \mathrm{dr}}$ (visualized on the $V$-w plane). $E$ : corresponding $I$ - $V$ curves in which the switch to class 2 excitability is paralleled by conversion of the steady-state $I$ - $V$ curve from nonmonotonic to monotonic.

steady-state $I$ - $V$ curve insofar as perithrehsold activation of $I_{\mathrm{K}, \mathrm{dr}}$ or $I_{\mathrm{M}}$ both cause that curve to become monotonic (Fig. 8E).

To summarize, MPOs are generated when inward current competes with slower-activating outward current. We originally attributed MPOs to the competition between $I_{\mathrm{Na}}$ and $I_{\mathrm{K}, \mathrm{dr}}$, but subsequent analysis suggested that $\theta$ frequency MPOs arise from competition between $I_{\mathrm{Na}}$ and an outward current slower than $I_{\mathrm{K}, \mathrm{dr}}$, namely $I_{\mathrm{M}}$. The competition between $I_{\mathrm{Na}}$ and $I_{\mathrm{M}}$ is the same as that between $I_{\mathrm{Na}}$ and $I_{\mathrm{K} . \mathrm{dr}}$ : initial activation of inward current is followed by delayed activation of outward current-this is manifested as spiraling on the respective phase plane. Interestingly, our results show that $I_{\mathrm{M}}$ can, theoretically, contribute to oscillations in different ways: by competing directly with $I_{\mathrm{Na}}$ as described above (thus producing $\theta$ frequency MPOs) and also by providing a sustained outward current that shifts threshold and thus modulates the competition between $I_{\mathrm{Na}}$ and $I_{\mathrm{K}, \mathrm{dr}}$ (thus producing higher frequency MPOs; see Fig. 3).

Our experimental data from CA1 pyramidal neurons argue that $I_{\mathrm{M}}$ functioned uniquely through the first mechanism. First, power spectral analysis revealed MPOs in the $\theta$ frequency range but not at higher frequencies (see Fig. 7). Second, adaptation caused an increase in $f_{\min }$ to between 10 and 30 spike/s, depending on shunting (see Fig. 4); this is high enough to allow $\theta$ frequency MPOs (see Fig. 5) and is therefore consistent with $f_{\min }$ being determined by competition between $I_{\mathrm{Na}}$ and $I_{\mathrm{M}}$ but it is lower than would be predicted if $f_{\min }$ was determined by competition between $I_{\mathrm{Na}}$ and $I_{\mathrm{K}, \mathrm{dr}}$. By exten- sion, we would therefore argue that CA1 pyramidal neurons convert from class 1 to class 2 excitability primarily because of the outward current contributed by $I_{\mathrm{M}}$ rather than because of the outward current contributed by $I_{\mathrm{K}, \mathrm{dr}}$ as initially hypothesized in Fig. 3. Importantly, voltage dependency of $I_{\mathrm{M}}$ is such that $I_{\mathrm{M}}$ can be activated at subthreshold voltages; by extension, a shift in $V^{*}$ can significantly modulate the strength of perithreshold activation (Fig. 9A), which has already been shown to have important functional consequences (Prescott et al. 2006).

\section{Effects of voltage threshold ( $\left.V^{*}\right)$ on strength of $I_{M}$}

Variable activation of $I_{\mathrm{M}}$ (based on modulation of $V^{*}$ ) is important for explaining certain aspects of our experimental results. For one, CA1 pyramidal neurons spiked at low rates on initial depolarization from rest (Fig. 4, $B$ and $C$ ); although $I_{\mathrm{M}}$ is slow to activate, it should be able prevent spiking below $\theta$ frequency (see preceding text). These data therefore suggest that $I_{\mathrm{M}}$ was not strongly activated on initial depolarization in nonshunted CA1 pyramidal neurons. This is consistent with the absence of resonance when nonshunted neurons were transiently depolarized to perithreshold voltages (Fig. 7D). However, nonshunted CA1 neurons clearly oscillated/resonated during sustained perithreshold depolarization (Fig. 7, $A$ and $C$ ), which indicates that $I_{\mathrm{M}}$ did eventually activate under those conditions. Delayed activation of $I_{\mathrm{M}}$ is consistent with the observation that adaptation increased $f_{\min }$ to $>\theta$ frequency (Fig. 4, $D$ and $E$ ). One likely explanation for delayed activation 
A $I_{M}$
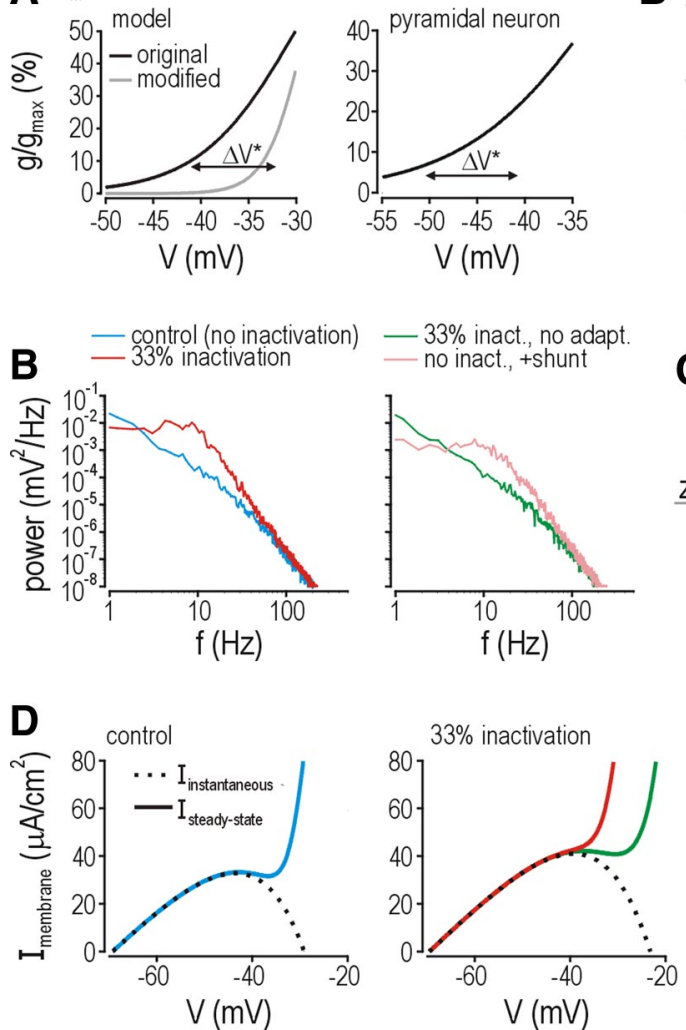

B

experiment
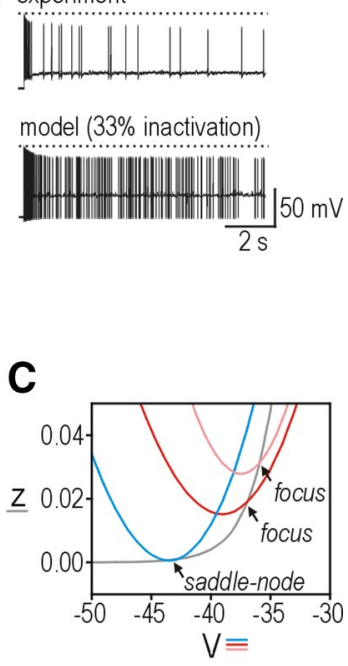

FIG. 9. Modulation of $I_{\mathrm{M}}$ via changes in $V^{*}$ and its implications for MPOs. A: voltage-dependent activation curve for $I_{\mathrm{M}}$ in our model (left; original: $\beta_{\mathrm{z}}=$ $-30 \mathrm{mV}$ and $\gamma_{\mathrm{z}}=5 \mathrm{mV}$; modified: $\beta_{\mathrm{z}}=-29 \mathrm{mV}$ and $\gamma_{\mathrm{z}}=2 \mathrm{mV} ; \tau_{\mathrm{z}}=400 \mathrm{~ms}$ and $\bar{g}_{\mathrm{M}}=2 \mathrm{mS} / \mathrm{cm}^{2}$ in both) and based on measurements from CA1 pyramidal neurons (Wu et al. 2008) (right). A depolarizing shift in spike threshold $\left(V^{*}\right)$ allows for increased subthreshold activation of $I_{\mathrm{M}}$. $B$ : slow decrement in spike amplitude observed experimentally (top) suggests that sodium channels slowly inactivate during sustained depolarization. This was reproduced in the model neuron by incorporating sodium channel inactivation implemented by multiplying $\bar{g}_{\mathrm{Na}}$ by $h$ where $\alpha h / \alpha \mathrm{t}=\frac{h_{\infty}(V)-h}{\tau_{h}}, h_{\infty}(V)=1-\frac{\alpha_{h}}{1+e^{\left(\beta_{h}-V\right) / \gamma_{h}}}, \alpha_{h}$ $=0.67, \beta_{\mathrm{h}}=-40 \mathrm{mV}$, and $\gamma_{\mathrm{h}}=8 \mathrm{mV}$, and $\tau_{\mathrm{h}}=1 \mathrm{~s} .33 \%$ inactivation of $g_{\mathrm{Na}}$ caused a $\sim 15 \%$ drop in spike amplitude (bottom), which is similar to that observed experimentally (top). The model here used $\bar{g}_{\mathrm{Na}}=24 \mathrm{mS} / \mathrm{cm}^{2}, \bar{g}_{\mathrm{K}, \mathrm{dr}}=30 \mathrm{mS} / \mathrm{cm}^{2}, \gamma_{\mathrm{w}}=8 \mathrm{mV}, \phi_{\mathrm{w}}=0.25$, and the modified $I_{\mathrm{M}}$ parameters reported in $A$. For subsequent simulations, steady-state inactivation was implemented simply by reducing $\bar{g}_{\mathrm{Na}}$ by $33 \%$. $C$ : power spectra demonstrate that the model neuron described in $B$ oscillated only when $V^{*}$ was depolarized by shunting (pink) or by sodium channel inactivation (red); compare with lack of oscillations under control conditions (i.e., no inactivation and no shunting; blue) or when $I_{\mathrm{M}}$ was removed (green). $D$ : phase plane showing intersection of $V$ - and $z$-nullclines. As predicted, the fixed point was converted from a saddle-node to a focus by both shunting and sodium channel inactivation, consistent with the conditions under which the model oscillated in $C$. E: shunting and sodium channel inactivation caused increased perithreshold activation of $I_{\mathrm{M}}$, which caused the steady-state $I-V$ curve to become monotonic, consistent with the change in phase plane geometry in $D$.

of $I_{\mathrm{M}}$ is that an even slower process, such as cumulative sodium channel inactivation that develops on the time scale of seconds (Fleidervish and Gutnick 1996), allows for increased activation of $I_{\mathrm{M}}$ when the neuron experiences sustained depolarization. Sodium channel inactivation was evident in our experimental data, for example, based on slow decrement in spike amplitude during sustained depolarization (Fig. 9B). Other results suggest that shunting allows for increased perithreshold activation of $I_{\mathrm{M}}$ : transient depolarizations caused $\theta$ frequency resonance (Fig. 7D) and $f_{\min }$ was $>\theta$ frequency on initial depolarization (Fig. $4, B$ and $C$ ) in shunted neurons. These data therefore suggest that shunting and cumulative sodium channel inactivation (or some other very slow process triggered by sustained depolarization) allow for increased perithreshold activation of $I_{\mathrm{M}}$ that causes net steady-state current to become outward, thus converting CA1 pyramidal neurons from class 1 to class 2 excitability and allowing them to oscillate at $\theta$ frequency.

To demonstrate the feasibility of this explanation, Fig. $9 \mathrm{C}$ shows how a model neuron with $I_{\mathrm{M}}$ oscillated at $\theta$ frequency when shunted or when sodium current was partially inacti- vated, whereas the same neuron did not oscillate (because $I_{\mathrm{M}}$ was not strongly activated below $V^{*}$ ) under control conditions (i.e., no inactivation or shunting). Removing $I_{\mathrm{M}}$ from the model neuron prevented sodium channel inactivation (Fig. 9C, green curve) or shunting (data not shown) from causing oscillations, thus confirming that these two forms of modulation affected $\theta$ frequency MPOs by modulating activation of $I_{\mathrm{M}}$ rather than by modulating activation of $I_{\mathrm{K}, \mathrm{dr}}$. Consistent with causing increased perithreshold activation of $I_{\mathrm{M}}$, shunting and sodium channel inactivation converted the intersection between the $V$ and $z$-nullclines from a saddle-node to a focus (Fig. 9D) and made the steady-state $I$ - $V$ curve monotonic (Fig. $9 E$ ).

\section{I S C U S S I O N}

In this study, we used computational modeling and experiments in which in vivo-like conditions were recreated in an acute slice preparation to investigate whether CA1 pyramidal neurons behave as integrators or resonators, i.e., exhibit class 1 or 2 excitability according to Hodgkin's (1948) classification 
scheme. Based on changes in voltage threshold, shape of the $f-I$ curve, and ability to oscillate/resonate, our results demonstrate that although pyramidal neurons behave as integrators under typical in vitro conditions, those same neurons behave as resonators when shunted and/or tonically depolarized as is likely to occur in vivo (see INTRODUCTION). The change in coding strategy is fully accounted for by the relative increase in outward current caused by increased activation of $I_{\mathrm{M}}$, and the effects of that outward current on spike initiation.

The switch in excitability is interesting for several reasons. First, spike-initiating dynamics are typically thought of as an intrinsic property of the neuron, but results here indicate that spike initiation can be influenced by extrinsic factors like synaptic input (see also Steriade 2001). Second, the spikeinitiating mechanism is mutable rather than being a fixed property of the neuron as evidenced by its qualitative change from SNIC bifurcation to Hopf bifurcation. But despite the qualitative change in bifurcation mechanism, both $f_{\min }$ and MPO frequency grow continuously from $0 \mathrm{~Hz}$ as the neuron transitions from class 1 to class 2 excitability (i.e., as net current at perithreshold potentials becomes more strongly outward). In fact, $f_{\min }$ and MPO frequency are directly related insofar as MPO frequency is less than $f_{\text {min }}+\Delta f$, where $\Delta f$ accounts for subthreshold MPOs occurring in the bistable region of the bifurcation diagram (see RESUlTS). Consistent with this, $\theta$ frequency $(3-10 \mathrm{~Hz})$ is less than our estimated value of $f_{\min }$ under in vivo conditions $(\sim 15-20 \mathrm{~Hz})$. This relationship between $f_{\min }$ and MPO frequency seems not to be broadly appreciated, but it suggests, for instance, that interneurons oscillating at $\gamma$ frequency $(30-80 \mathrm{~Hz})$ must have even higher $f_{\min }$, which appears to be the case (Erisir et al. 1999; Tateno et al. 2004).

Several studies have documented the importance of intrinsic membrane properties for network synchronization (Di Garbo et al. 2007; Geisler et al. 2005; Pfeuty et al. 2003; Tateno and Robinson 2007). Pfeuty et al. (2003) specifically concluded that potassium currents promote synchrony, whereas persistent sodium current impedes synchrony, which is consistent with our results insofar as outward current encourages class 2 excitability, whereas inward current encourages class 1 excitability. Golomb et al. (2007) recently showed that increasing the sodium window current (which contributes inward current at perithreshold potentials) changed the bifurcation mechanism and prevented subthreshold voltage fluctuations, consistent with inward current switching the excitability of their model neuron to class 1 . Hutcheon et al. (1996) also showed through modeling that shunting could encourage resonance, although they attributed that effect to a quantitative change in the membrane time constant rather than a qualitative change in spike-initiating mechanism. Our results are also consistent with work showing that adaptation mediated by $I_{\mathrm{M}}$ encourages class 2 excitability (Ermentrout et al. 2001), whereas cholinergic agonists, which block $I_{\mathrm{M}}$, have the opposite effect (Stiefel et al. 2008). Moreover, these results are consistent with reported effects of acetylcholine on low-frequency oscillations during slow-wave sleep (Steriade 2004). At first glance, our results appear to contradict Fernandez and White (2008), who showed that MPOs in entorhinal stellate cells were strongly attenuated by increased membrane conductance; in fact, our results are consistent insofar as shunting also attenuated MPOs in CA1 pyramidal cells and abolished spiking associated with those
MPOs (see Fig. 7A). One must consider that, to oscillate, CA1 pyramidal cells require a depolarizing shift in voltage threshold (caused by shunting, for example) to allow sufficient subthreshold activation of $I_{M}$ for the neuron to convert to class 2 excitability; entorhinal stellate cells, on the other hand, are likely class 2 under "control" conditions. Therefore although shunting is required for oscillations in the former cell type, it is not required for oscillations in the latter.

In the initial stages of this study, we hypothesized that shunting and adaptation (mediated by $I_{\mathrm{M}}$ ) allowed for increased activation of the delayed rectifier potassium current $I_{\mathrm{K}, \mathrm{dr}}$ and that increased perithreshold activation of $I_{\mathrm{K}, \mathrm{dr}}$ was responsible for making net steady-state current outward, thus causing excitability to become class 2 . However, although increased perithreshold activation of $I_{\mathrm{K}, \mathrm{dr}}$ could theoretically contribute to the generation of high (e.g., $\gamma$ ) frequency oscillations, subsequent analysis (see Fig. 8) indicated that $I_{\mathrm{K}, \mathrm{dr}}$ did not have the kinetics required to generate low, $\theta$ frequency oscillations. This result is, perhaps, quite obvious. Nonetheless, results of that initial analysis provided the insight necessary to explain differential activation of $I_{\mathrm{M}}$ depending on operating conditions. Perithreshold activation of $I_{M}$ can be increased by the same depolarizing shift in voltage threshold that could increase perithreshold activation of $I_{\mathrm{K}, \mathrm{dr}}$. Perithreshold activation of outward currents like $I_{\mathrm{M}}$ contribute to the shift in threshold, but, more important for understanding modulation of perithreshold activation of $I_{\mathrm{M}}$ are those processes that act on a faster or slower time scale than $I_{M}$. Shunting and cumulative sodium channel inactivation caused by tonic depolarization are two such processes that cause a depolarizing shift in voltage threshold. Both are liable to be prominent under in vivo conditions but are modest under in vitro conditions, meaning $I_{\mathrm{M}}$ is liable to have a less pronounced effect in vitro than it would have in vivo. The result is that CA1 pyramidal neurons that function as integrators (class 1 excitable) in slice experiments may function as resonators (class 2 excitable) in the intact, awake brain. This is important given that neuron models are usually built based on parameters measured in vitro; such models may fail to reproduce, even qualitatively, important phenomena like oscillations and resonance. For one, the classic leaky integrate-and-fire model may not be ideal for modeling pyramidal cells, although variations such as the exponential integrate-and-fire model seem to capture some of the nonlinear dynamics at spike threshold (e.g., Fourcaud-Trocme et al. 2003).

To conclude, the dynamical mechanism underlying spike initiation can change qualitatively depending on conditions such as the level of background synaptic input. A switch in spike-initiating mechanism has several computationally important consequences and adds to a growing list of potential difficulties in extrapolating from in vitro data to explain neuronal operation in vivo. Nonetheless, in vivo-like conditions can be recreated in vitro and represent a useful approach for resolving potential discrepancies. These results remind us that vigilance is necessary when using a reductionist approach that relies on experimental conditions (e.g., brain slices) that do not fully replicate the complexities of the intact brain. Model building and carefully designed experiments are necessary to recognize and surmount these shortcomings. 


\section{A C K N O W LED G MENTS}

We thank D. Spencer for helpful comments on the manuscript. Present address of S. Ratté: Dept. of Neurobiology, University of Pittsburgh, 200 Lothrop St., Pittsburgh, PA 15213.

\section{G R A N T S}

This research was supported by funding from the Howard Hughes Medical Institute to T. J. Sejnowksi and the Natural Sciences and Engineering Research Council of Canada to Y. De Koninck. S. A. Prescott was a long-term fellow with the Human Frontier Science Program, and Y. De Koninck is a Chercheur National with the Fonds de la Recherche en Santé du Québec.

\section{REFERENCES}

Anderson JS, Lampl I, Gillespie DC, Ferster D. The contribution of noise to contrast invariance of orientation tuning in cat visual cortex. Science 290: 1968-1972, 2000.

Barry PH, Lynch JW. Liquid junction potentials and small cell effects in patch-clamp analysis. J Membr Biol 121: 101-117, 1991.

Bernander Ö, Douglas RJ, Martin KA, Koch C. Synaptic background activity influences spatiotemporal integration in single pyramidal cells. Proc Natl Acad Sci USA 88: 11569-11573, 1991.

Borisyuk A, Rinzel J. Understanding neuronal dynamics by geometrical dissection of minimal models. In: Methods and Models in Neurophysics, Proc Les Houches Summer School, edited by Chow C, Gutkin B, Hansel D, Meunier C; Dalibard J. Amsterdam: Elsevier, 2005, p. 19-72.

Buzsáki G. Rhythms of the Brain. Oxford, UK: Oxford Univ. Press, 2006.

Chance FS, Abbott LF, Reyes AD. Gain modulation from background synaptic input. Neuron 35: 773-782, 2002.

Destexhe A, Paré D. Impact of network activity on the integrative properties of neocortical pyramidal neurons in vivo. $J$ Neurophysiol 81: 1531-1547, 1999.

Destexhe A, Rudolph M, Fellous JM, Sejnowski TJ. Fluctuating synaptic conductances recreate in vivo-like activity in neocortical neurons. Neuroscience 107: 13-24, 2001.

Destexhe A, Rudolph M, Pare D. The high-conductance state of neocortical neurons in vivo. Nat Rev Neurosci 4: 739-751, 2003.

Di Garbo A, Barbi M, Chillemi S. The synchronization properties of a network of inhibitory interneurons depend on the biophysical model. Biosystems 88: 216-227, 2007.

Dorval AD, White JA. Channel noise is essential for perithreshold oscillations in entorhinal stellate neurons. $J$ Neurosci 25: 10025-10028, 2005.

Erchova I, Kreck G, Heinemann U, Herz AV. Dynamics of rat entorhinal cortex layer II and III cells: characteristics of membrane potential resonance at rest predict oscillation properties near threshold. J Physiol 560: 89-110, 2004.

Erisir A, Lau D, Rudy B, Leonard CS. Function of specific $\mathrm{K}^{+}$channels in sustained high-frequency firing of fast-spiking neocortical interneurons. J Neurophysiol 82: 2476-2489, 1999.

Ermentrout B. Type I membranes, phase resetting curves, and synchrony. Neural Comput 8: 979-1001, 1996.

Ermentrout B, Pascal M, Gutkin B. The effects of spike frequency adaptation and negative feedback on the synchronization of neural oscillators. Neural Comput 13: 1285-1310, 2001.

Fernandez FR, White JA. Artificial synaptic conductances reduce subthreshold oscillations and periodic firing in stellate cells of the entorhinal cortex. J Neurosci 28: 3790-3803, 2008.

Fleidervish IA, Gutnick MJ. Kinetics of slow inactivation of persistent sodium current in layer $\mathrm{V}$ neurons of mouse neocortical slices. $J$ Neurophysiol 76: 2125-2130, 1996.

Fourcaud-Trocme N, Hansel D, van Vreeswijk C, Brunel N. How spike generation mechanisms determine the neuronal response to fluctuating inputs. J Neurosci 23: 11628-11640, 2003.

Geisler C, Brunel N, Wang XJ. Contributions of intrinsic membrane dynamics to fast network oscillations with irregular neuronal discharges. $\mathrm{J} \mathrm{Neu}$ rophysiol 94: 4344-4361, 2005.

Gillespie DT. The mathematics of Brownian Motion and Johnson noise. Am J Phys 64: 225-240, 1996.

Golomb D, Donner K, Shacham L, Shlosberg D, Amitai Y, Hansel D. Mechanisms of firing patterns in fast-spiking cortical interneurons. PLoS Comput Biol 3: e156, 2007.

Gustafsson B, Wigstrom H. Shape of frequency-current curves in CAI pyramidal cells in the hippocampus. Brain Res 223: 417-421, 1981.
Hansel D, Mato G, Meunier C. Synchrony in excitatory neural networks. Neural Comput 7: 307-337, 1995.

Harris KD, Henze DA, Hirase H, Leinekugel X, Dragoi G, Czurko A, Buzsáki G. Spike train dynamics predicts theta-related phase precession in hippocampal pyramidal cells. Nature 417: 738-741, 2002.

Hodgkin AL. The local electric changes associated with repetitive action in a non-medullated axon. J Physiol 107: 165-181, 1948.

Hu H, Vervaeke K, Storm JF. Two forms of electrical resonance at theta frequencies, generated by $\mathrm{M}$-current, $\mathrm{h}$-current and persistent $\mathrm{Na}^{+}$current in rat hippocampal pyramidal cells. J Physiol 545: 783-805, 2002.

Hutcheon B, Miura RM, Puil E. Models of subthreshold membrane resonance in neocortical neurons. J Neurophysiol 76: 698-714, 1996.

Hutcheon B, Yarom Y. Resonance, oscillation and the intrinsic frequency preferences of neurons. Trends Neurosci 23: 216-222, 2000.

Izhikevich EM. Dynamical Systems in Neuroscience. Cambridge, MA: MIT Press, 2007.

Kaplan D and Glass L. Understanding Nonlinear Dynamics. New York: Springer-Verlag, 1995.

Kepler TB, Abbott LF, Marder E. Reduction of conductance-based neuron models. Biol Cybern 66: 381-387, 1992.

Lampl I, Yarom Y. Subthreshold oscillations and resonant behavior: two manifestations of the same mechanism. Neuroscience 78: 325-341, 1997.

Leung LS, Yu HW. Theta-frequency resonance in hippocampal CA1 neurons in vitro demonstrated by sinusoidal current injection. J Neurophysiol 79: 1592-1596, 1998.

Leung LW, Yim CY. Intrinsic membrane potential oscillations in hippocampal neurons in vitro. Brain Res 553: 261-274, 1991.

Morris C, Lecar H. Voltage oscillations in the barnacle giant muscle fiber. Biophys J 35: 193-213, 1981.

Paré D, Shink E, Gaudreau H, Destexhe A, Lang EJ. Impact of spontaneous synaptic activity on the resting properties of cat neocortical pyramidal neurons in vivo. J Neurophysiol 79: 1450-1460, 1998.

Pfeuty B, Mato G, Golomb D, Hansel D. Electrical synapses and synchrony: the role of intrinsic currents. J Neurosci 23: 6280-6294, 2003.

Pike FG, Goddard RS, Suckling JM, Ganter P, Kasthuri N, Paulsen O. Distinct frequency preferences of different types of rat hippocampal neurones in response to oscillatory input currents. J Physiol 529: 205-213, 2000.

Pinto RD, Elson RC, Szucs A, Rabinovich MI, Selverston AI, Abarbanel HD. Extended dynamic clamp: controlling up to four neurons using a single desktop computer and interface. J Neurosci Methods 108: 39-48, 2001.

Prescott SA, De Koninck Y. Gain control of firing rate by shunting inhibition: roles of synaptic noise and dendritic saturation. Proc Natl Acad Sci USA 100: 2076-2081, 2003.

Prescott SA, De Koninck Y. Impact of background synaptic activity on neuronal response properties revealed by stepwise replication of in vivo-like conditions in vitro. In: The Dynamic Clamp: From Principles to Applications, edited by Destexhe A, Bal T. Berlin: Springer. In press.

Prescott SA, De Koninck Y, Sejnowski TJ. Biophysical basis for three distinct dynamical mechanisms of action potential initiation. PLoS Comput Biol 4: el000198, 2008.

Prescott SA, Ratté S, De Koninck Y, Sejnowski TJ. Nonlinear interaction between shunting and adaptation controls a switch between integration and coincidence detection in pyramidal neurons. J Neurosci 26: 9084-9097, 2006.

Rinzel J, Ermentrout GB. Analysis of neural excitability and oscillations. In: Methods in Neuronal Modeling: From Ions to Networks, edited by Koch C, Segev I. Cambridge, MA: The MIT Press, 1998, p. 251-291.

Rudolph M, Destexhe A. Tuning neocortical pyramidal neurons between integrators and coincidence detectors. J Comput Neurosci 14: 239-251, 2003.

Schreiber S, Erchova I, Heinemann U, Herz AV. Subthreshold resonance explains the frequency-dependent integration of periodic as well as random stimuli in the entorhinal cortex. J Neurophysiol 92: 408-415, 2004.

Steriade M. Impact of network activities on neuronal properties in corticothalamic systems. J Neurophysiol 86: 1-39, 2001.

Steriade M. Acetylcholine systems and rhythmic activities during the wakingsleep cycle. Prog Brain Res 145: 179-196, 2004.

Stiefel KM, Gutkin B, Sejnowski TJ. The effects of cholinergic neuromodulation on neuronal phase-response curves of modeled cortical neurons. J Comput Neurosci In press. 
Strogatz SH. Nonlinear Dynamics and Chaos: With Applications to Physics, Biology, Chemistry, and Engineering. Don Mills, ON: Addison-Wesley, 1998.

Tateno T, Harsch A, Robinson HP. Threshold firing frequency-current relationships of neurons in rat somatosensory cortex: type 1 and type 2 dynamics. J Neurophysiol 92: 2283-2294, 2004.

Tateno T, Robinson HP. Quantifying noise-induced stability of a cortical fast-spiking cell model with Kv3-channel-like current. Biosystems 89: 110116, 2007.

Traub RD, Miles R. Neuronal Networks of the Hippocampus. Cambridge, UK: Cambridge Univ. Press, 1991.
Wang XJ, Buzsáki G. Gamma oscillation by synaptic inhibition in a hippocampal interneuronal network model. J Neurosci 16: 6402-6413, 1996.

Wilson HR. Simplified dynamics of human and mammalian neocortical neurons. J Theor Biol 200: 375-388, 1999.

Wolfart J, Debay D, Le Masson G, Destexhe A, Bal T. Synaptic background activity controls spike transfer from thalamus to cortex. Nat Neurosci 8: 1760-1767, 2005.

Wu WW, Chan CS, Surmeier DJ, Disterhoft JF. Coupling of L-type $\mathrm{Ca}^{2+}$ channels to KV7/KCNQ channels creates a novel, activity-dependent, homeostatic intrinsic plasticity. J Neurophysiol 100: 1897-1908, 2008. 